\title{
THE COMPLEXITY OF CHROMATIC STRENGTH AND CHROMATIC EDGE STRENGTH
}

\author{
DÁNIEL MARX
}

\begin{abstract}
The sum of a coloring is the sum of the colors assigned to the vertices (assuming that the colors are positive integers). The sum $\Sigma(G)$ of graph $G$ is the smallest sum that can be achieved by a proper vertex coloring of $G$. The chromatic strength $s(G)$ of $G$ is the minimum number of colors that is required by a coloring with sum $\Sigma(G)$. For every $k$, we determine the complexity of the question "Is $s(G) \leq k$ ?": it is coNP-complete for $k=2$ and $\Theta_{2}^{p}$-complete for every fixed $k \geq 3$. We also study the complexity of the edge coloring version of the problem, with analogous definitions for the edge sum $\Sigma^{\prime}(G)$ and the chromatic edge strength $s^{\prime}(G)$. We show that for every $k \geq 3$, it is $\Theta_{2}^{p}$-complete to decide whether $s^{\prime}(G) \leq k$ holds. As a first step of the proof, we present graphs for every $r \geq 3$ with chromatic index $r$ and edge strength $r+1$. For some values of $r$, such graphs were not known before.

Keywords. graph coloring, chromatic strength, chromatic number, chromatic index
\end{abstract}

Subject classification. 68Q17

\section{Introduction}

A vertex coloring of graph $G(V, E)$ is an assignment $\psi: V \rightarrow \mathbb{N}$ of colors (positive integers) to the vertices such that adjacent vertices receive different colors. The sum of a vertex coloring $\psi$ is the sum of the colors assigned to the vertices, $\Sigma_{\psi}(G)=\sum_{v \in V} \psi(v)$. The chromatic sum $\Sigma(G)$ of graph $G$ is the smallest sum that a proper coloring of $G$ can have. Edge coloring versions of the above concepts are defined analogously, the sum of an edge coloring $\psi$ is denoted by $\Sigma_{\psi}^{\prime}(G)$, while the chromatic edge sum of $G$ is $\Sigma^{\prime}(G)$.

In the minimum sum coloring problem our aim is to find a coloring with sum as small as possible, that is, to determine the chromatic sum of the graph. The problem was first studied independently by Supowit (1987) and by Kubicka \& Schwenk (1989) (see also Kubicka 1989). Minimum sum coloring is motivated by applications in scheduling and VLSI design (see e.g., Bar-Noy 
et al. 1998 and Nicoloso et al. 1999). Determining the chromatic sum is NPhard in general. In fact, it remains hard also on some classes of graphs where coloring is easy, such as bipartite graphs (Bar-Noy \& Kortsarz 1998) and interval graphs (Marx 2005; Szkaliczki 1999). Approximation algorithms were given for several graph classes: the minimum sum can be 4-approximated in perfect graphs (Bar-Noy et al. 1998), 1.796-approximated in interval graphs (Halldórsson et al. 2003), and 27/26-approximated in bipartite graphs (Giaro et al. 2002). Considering the analogous minimum sum edge coloring problem, determining the chromatic edge sum is NP-hard even for bipartite graphs (Giaro \& Kubale 2000), but can be solved in polynomial time for trees (Giaro $\&$ Kubale 2000; Salavatipour 2003). Moreover, there is a 1.796-approximation algorithm for bipartite graphs (Halldórsson et al. 2003) and a 2-approximation algorithm for general graphs (Bar-Noy et al. 1998).

Kubicka \& Schwenk (1989) noted that the number of colors required by a minimum sum coloring can be much greater than the chromatic number of the graph. In particular, for every $k \geq 2$, they show a tree for which every minimum sum coloring uses at least $k$ different colors (see Figure 1.1 for an example of the case $k=3$ ). Let $s(G)$ be the chromatic strength of $G$, which is the smallest number of colors required in a minimum sum coloring of $G$. The chromatic edge strength $s^{\prime}(G)$ is defined analogously. Clearly, $s(G) \geq \chi(G)$, but as the example above shows, $s(G)-\chi(G)$ can be arbitrarily large. On the other hand, Mitchem et al. (1997) and independently Hajiabolhassan et al. (2000) proved an analog of Vizing's Theorem showing that $s^{\prime}(G) \leq \Delta(G)+1$ in every simple graph $G$. Hence we have

$$
\Delta(G) \leq \chi^{\prime}(G) \leq s^{\prime}(G) \leq \Delta(G)+1
$$

if $G$ is a simple graph. Harary and Plantholt conjectured (see West (Winter 1994-95)) that the second inequality is in fact an equality, hence if a simple graph is $k$-edge-colorable, then it has a minimum sum edge coloring with $k$ colors. However, this conjecture turned out to be false: for every odd integer $k \geq 5$, a graph with chromatic index $k$ and edge strength $k+1$ was given in Mitchem et al. (1997). Moreover, Hajiabolhassan et al. (2000) gives such a graph for $k=4$. Thus we can conclude that the chromatic index and the chromatic edge strength are not always the same.

Here we study the computational complexity of determining the chromatic strength and chromatic edge strength of a simple graph. The complexity of the vertex strength is investigated in Salavatipour (2003):

Theorem 1.1 (Salavatipour 2003). For every $k \geq 3$, it is NP-hard to decide whether $s(G) \leq k$ holds for a given graph $G$. 


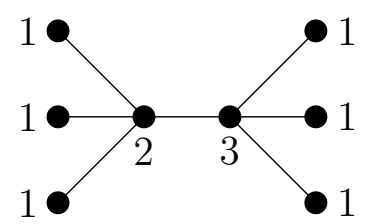

Figure 1.1: A tree with strength 3. The figure shows a minimum sum coloring with sum 11, while every 2-coloring has sum 12 .

Notice that it is not clear whether this problem belongs to NP. A minimum sum $k$-coloring is not a good certificate for $s(G) \leq k$, since we cannot verify that it is indeed a minimum sum coloring. On the other hand, the problem does not seem to belong to coNP either: a minimum sum coloring with more than $k$ colors does not certify that $s(G)>k$, since it does not prove that this sum cannot be achieved using only $k$ colors. Our main contribution is that we determine the exact complexity of the chromatic strength problem by showing that for every $k \geq 3$, it is $\Theta_{2}^{p}$-complete to decide whether $s(G) \leq k$ holds. The class $\Theta_{2}^{p}$ contains those problems that can be solved in polynomial time with a logarithmic number of NP oracle calls (see Section 5 for definitions). It is interesting to see a natural coloring problem that is complete for this lesserknown complexity class. In Salavatipour (2003) the complexity of the case $k=2$ was left as an open question. We answer this question by showing that deciding $s(G) \leq 2$ is coNP-complete.

We obtain our $\Theta_{2}^{p}$-completeness result for the chromatic strength by proving the stronger statement that even the more restricted chromatic edge strength problem is $\Theta_{2}^{p}$-complete. The complexity of edge strength is also treated in Salavatipour (2003). By observing that $s^{\prime}(G)=\chi^{\prime}(G)$ for every regular simple graph, they conclude that for regular graphs "Is $s^{\prime}(G) \leq k$ ?" has the same complexity as "Is $\chi^{\prime}(G) \leq k$ ?" and the latter problem is known to be NPcomplete for every $k \geq 3$ (Holyer 1981; Leven \& Galil 1983). However, if we want to prove that edge strength is $\Theta_{2}^{p}$-complete (that is, harder than the chromatic index problem), then necessarily we have to consider graphs where the edge strength and the chromatic index are not the same. Therefore, we need substantially different (and more complicated) arguments than in Salavatipour (2003).

We prove the $\Theta_{2}^{p}$-completeness of chromatic edge strength the following way. First we show that for every $k \geq 3$, there is a simple graph $G_{k}$ with $\Delta\left(G_{k}\right)=$ $\chi^{\prime}\left(G_{k}\right)=k$ and $s^{\prime}\left(G_{k}\right)=k+1$. That is, we give counterexamples to the conjecture of Harary and Plantholt in all the remaining cases. Next, in Section 5 
we introduce some new $\Theta_{2}^{p}$-complete problems, which might be of independent interest as well. In particular, we show that it is $\Theta_{2}^{p}$-complete to decide whether every minimum vertex cover of a given graph includes the distinguished vertex $\hat{v}$. Finally, we show that if there is a graph with maximal degree $k$ where the chromatic index and the chromatic edge strength are different, then it is $\Theta_{2^{-}}^{p}$ complete to decide whether the edge strength is $k$ in a graph with maximum degree $k$. Together with the existence of the counterexample graphs, this gives the required result.

The paper is organized as follows. In Section 2, we show that it is coNPcomplete to decide whether $s(G) \leq 2$. In the rest of the paper, we consider only the edge coloring version of the problem. Section 3 introduces notation and tools for edge colorings. The counterexamples to the conjecture of Harary and Plantholt are given in Section 4. In Section 5 we summarize the results

on the complexity class $\Theta_{2}^{p}$, and introduce the new $\Theta_{2}^{p}$-complete problems. The reduction for the main hardness result is presented in Section 6. The construction of the key gadget of the reduction is given in Section 7 .

\section{Vertex strength of bipartite graphs}

In this section we prove that for $k=2$, it is coNP-complete to decide whether $s(G) \leq k$ holds. Notice that, unlike in the case $k \geq 3$, now it is easy to see that the problem is in coNP. First, the question makes sense only if the graph is bipartite, otherwise trivially $s(G) \geq 3$. In a bipartite graph the sum of the best 2-coloring is easy to determine: each connected component of the graph has exactly two 2-colorings, and taking the better coloring of each component gives the best 2-coloring of the graph. Therefore, a minimum sum coloring with more than 2 colors certifies that $s(G) \leq 2$ does not hold: one can determine the sum of the best 2-coloring, and check that it is indeed larger than the sum of the given coloring. Thus the problem is in coNP.

The proof of coNP-hardness is by reduction from the precoloring extension problem. Precoloring extension (PREXT) is a generalization of vertex coloring (Tuza 1997): we are given a graph $G(V, E)$ with a subset $W \subseteq V$ of vertices having preassigned colors, the question is whether this precoloring can be extended to a proper $k$-coloring of the graph. We denote by 1-PRExT the special case where every color is used at most once in the precoloring. 1-PRExT is NP-complete for bipartite graphs (Hujter \& Tuza 1993), but polynomial-time solvable for interval graphs (Biró et al. 1992) and more generally, for chordal graphs (Marx 2004). In our proof, we need the following result: 


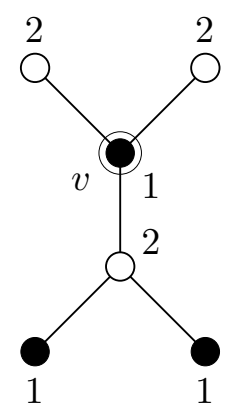

(a)

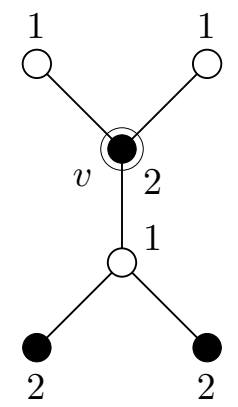

(b)

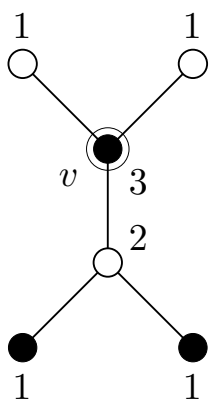

(c)

Figure 2.1: The new vertices attached to vertex $v$ of $H$, and three minimum sum colorings that assign to vertex $v$ (a) color 1, (b) color 2, (c) color 3.

TheOrem 2.1 (Bodlaender et al. 1994). 1-PRExT is NP-complete for bipartite graphs, even if the number of colors is 3 .

Moreover, it can be assumed that the 3 precolored vertices are in the same bipartition class (see the proof in Bodlaender et al. (1994)).

ThEOREM 2.2. Given a graph $G$, it is coNP-complete to decide if $s(G) \leq 2$ holds.

Proof. As we have noted above, the problem is in coNP. Hardness is proved by reduction from 1-PRExT for bipartite graphs. Given a bipartite graph $H(A, B ; E)$ with three precolored vertices $v_{1}, v_{2}, v_{3} \in A$, we construct a (bipartite) graph $G$ such that $s(G) \leq 2$ if and only if the precoloring of $H$ cannot be extended to the whole graph. We assume that vertex $v_{i}(i=1,2,3)$ is precolored with color $i$.

To construct the graph $G$, we attach 5 new vertices to every non-precolored vertex $v$ of $H$ (see Figure 2.1). Let the set $V_{v}$ contain vertex $v$ and the 5 vertices attached to it. In every coloring, the sum of the 6 vertices in $V_{v}$ is at least 9. Moreover, as shown in Figure 2.1a-c, this minimum sum 9 can be achieved with colorings that assign color 1,2 , or 3 to vertex $v$.

We attach 9 new vertices to the three precolored vertices $v_{1}, v_{2}, v_{3}$ (see Figure 2.2). Denote by $V^{*}$ the set of these 12 vertices. The vertices in the set $V^{*}$ have a sum of at least 17 in every coloring. Furthermore, it can be verified by inspection that the coloring shown in the figure is the unique minimum sum coloring of $V^{*}$. This completes the description of the graph $G$. Clearly, if $H$ is bipartite, then $G$ is bipartite as well. 


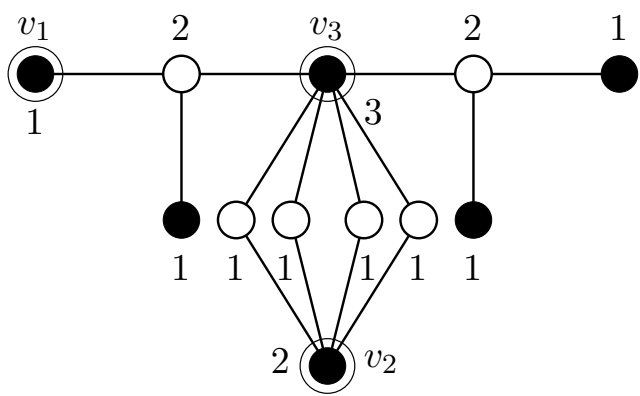

Figure 2.2: The graph that connects the precolored vertices $v_{1}, v_{2}, v_{3}$, and its unique minimum sum coloring with sum 17.

If the graph $H$ has $n+3$ vertices, then $G$ has $6 n+12$ vertices, and the sum of every coloring is at least $9 n+17$. Moreover, $G$ has a 2 -coloring with sum $9 n+18$ : color $v_{1}, v_{2}, v_{3}$, and their bipartition class with color 2 , the other class receives color 1 . Every set $V_{v}$ has sum 9 in this coloring, while $V^{*}$ has sum 18. This means that $s(G)>2$ if and only if there is a coloring of $G$ with sum exactly $9 n+17$ : otherwise the sum of $G$ is $9 n+18$, which can be also achieved by a 2 -coloring. If there is a coloring $\psi$ with sum $9 n+17$, then it induces a coloring of $H$. For such a coloring $\psi$, the sum of $\psi$ has to be exactly 17 on the vertices of $V^{*}$. Therefore, $V^{*}$ is colored as shown in Figure 2.2, thus the coloring induced by $\psi$ is a precoloring extension of $H$.

To prove the other direction, assume that $H$ has a precoloring extension with 3 colors. This coloring can be extended to a coloring of $G$ having sum $9 n+17$. In $V^{*}$, the coloring can be extended to the coloring shown in Figure 2.2. In every $V_{v}$, depending on the color of $v$, the coloring can be extended to one of the three colorings shown in Figure 2.1. The set $V^{*}$ has sum 17 in the resulting coloring, while every $V_{v}$ has sum 9 . Thus the sum of the coloring is $9 n+17$, and $s(G)=3$ follows.

\section{Minimum sum edge coloring}

For the rest of the paper, we consider only edge colorings, hence even if it is not noted explicitly, "coloring" will mean "edge coloring." We introduce notation and new parameters that turn out to be useful in studying minimum sum edge colorings. Let $\psi$ be an edge coloring of $G(V, E)$, and let $E_{v}$ be the set of edges incident to vertex $v$. For every $v \in V$, let $\Sigma_{\psi}^{\prime}(v)=\sum_{e \in E_{v}} \psi(e)$ be the sum of $v$, and for a subset $V^{\prime} \subseteq V$, let $\Sigma_{\psi}^{\prime}\left(V^{\prime}\right)=\sum_{v \in V^{\prime}} \Sigma_{\psi}^{\prime}(v)$. Clearly, $\Sigma_{\psi}^{\prime}(V)=2 \Sigma_{\psi}^{\prime}(G)$; therefore, minimizing $\Sigma_{\psi}^{\prime}(V)$ is equivalent to minimizing $\Sigma_{\psi}^{\prime}(G)$. 
The degree of vertex $v$ is denoted by $d(v):=\left|E_{v}\right|$. For every vertex $v$, let $\ell(v):=\sum_{i=1}^{d(v)} i=d(v)(d(v)+1) / 2$, and for a set of vertices $V^{\prime} \subseteq V$, let $\ell\left(V^{\prime}\right):=\sum_{v \in V^{\prime}} \ell(v)$. Since $\Sigma_{\psi}^{\prime}(v)$ is the sum of $d(v)$ distinct positive integers, $\Sigma_{\psi}^{\prime}(v) \geq \ell(v)$ in every proper coloring $\psi$. Let $\epsilon_{\psi}(v)=\Sigma_{\psi}^{\prime}(v)-\ell(v) \geq 0$ be the error of vertex $v$ in coloring $\psi$. For $V^{\prime} \subseteq V$ we define $\epsilon_{\psi}\left(V^{\prime}\right)=\sum_{v \in V^{\prime}} \epsilon_{\psi}(v)$, and call $\epsilon_{\psi}(V)$ the error of coloring $\psi$. The error is always non-negative: $\Sigma_{\psi}^{\prime}(V) \geq$ $\ell(V)$, hence $\epsilon_{\psi}(V)=\Sigma_{\psi}^{\prime}(V)-\ell(V) \geq 0$. Notice that $\epsilon_{\psi}(V)$ has the same parity for every coloring $\psi$. Minimizing the error of the coloring is clearly equivalent to minimizing the sum of the coloring. In particular, if $\psi$ is a zero error coloring, that is, $\epsilon_{\psi}(V)=0$, then $\psi$ is a minimum sum coloring of $G$. In a zero error coloring, the edges incident to vertex $v$ are colored with the colors $1,2, \ldots, d(v)$.

However, in general, $G$ does not necessarily have a zero error coloring. For every $V^{\prime} \subseteq V$, the error of $V^{\prime}$ is $\epsilon\left(V^{\prime}\right)=\min _{\psi} \epsilon_{\psi}\left(V^{\prime}\right)$, the smallest error $V^{\prime}$ can have in a proper coloring of $G$. (Notice that $\epsilon\left(V^{\prime}\right)=\sum_{v \in V^{\prime}} \epsilon(\{v\})$ does not always hold, in fact, $\epsilon(\{v\})=0$ for every $v \in V)$.

Quasigraphs. Parallel edges are not allowed for the graphs considered in this paper. However, for convenience we extend the problem by introducing half-loops. A half-loop is a loop that contributes only 1 to the degree of its end vertex. Every vertex has at most one half-loop. If a graph is allowed to have half-loops, then it will be called a quasigraph (the terminology half-loop and quasigraph is borrowed from Lovász 1997). In a quasigraph, the sum of an edge coloring is defined to be the sum of the color of the edges plus half the sum of the color of the half-loops; therefore, the sum of a quasigraph is not necessarily an integer. However, the error of a coloring is always integer, and with these definitions it remains true that the sum of the vertices is twice the sum of the edges.

The following observation shows that allowing half-loops does not make the problem more difficult:

Proposition 3.1. Given a quasigraph $G$, one can create in polynomial time a graph $G^{\prime}$ such that $\Sigma^{\prime}\left(G^{\prime}\right)=2 \Sigma^{\prime}(G)$ and $s^{\prime}\left(G^{\prime}\right)=s^{\prime}(G)$.

Proof. To obtain $G^{\prime}$, take two disjoint copies $G_{1}, G_{2}$ of $G$ and remove every half-loop. If there was a half-loop at $v$ in $G$, then add an edge $v_{1} v_{2}$ to $G^{\prime}$, where $v_{1}$ and $v_{2}$ are the vertices corresponding to $v$ in $G_{1}$ and $G_{2}$, respectively. In graph $G^{\prime}$, give to every edge the color of the corresponding edge in $G$. If the sum of the coloring in $G$ was $S$, then we obtain a coloring in $G^{\prime}$ with sum $2 S$ : 
two edges of $G^{\prime}$ correspond to every edge of $G$, but only one edge corresponds to every half-loop of $G$.

On the other hand, one can show that if $G^{\prime}$ has a $k$-coloring with sum $S$, then $G$ has a $k$-coloring with sum at most $S / 2$. The edges of $G^{\prime}$ can be partitioned into three sets $E_{1}, E_{2}, E^{\prime}$ : set $E_{i}$ contains the edges induced by $G_{i}(i=1,2)$, and $E^{\prime}$ contains the edges corresponding to the half-loops. If $\psi$ is an edge coloring of $G^{\prime}$ with sum $S$, then $S=\Sigma_{\psi}^{\prime}\left(E_{1}\right)+\Sigma_{\psi}^{\prime}\left(E_{2}\right)+\Sigma_{\psi}^{\prime}\left(E^{\prime}\right)$. Without loss of generality, it can be assumed that $\Sigma_{\psi}^{\prime}\left(E_{1}\right) \leq \Sigma_{\psi}^{\prime}\left(E_{2}\right)$, hence $\Sigma_{\psi}^{\prime}\left(E_{1}\right)+\Sigma_{\psi}^{\prime}\left(E^{\prime}\right) / 2 \leq S / 2$. The $k$-coloring of $G_{1}$ induced by $\psi$ has sum $\Sigma_{\psi}^{\prime}\left(E_{1}\right)+$ $\Sigma_{\psi}^{\prime}\left(E^{\prime}\right) / 2 \leq S / 2$, since the edges in $E^{\prime}$ correspond to half-loops.

Therefore, finding a minimum sum edge coloring for the quasigraph $G$ is the same problem as finding a minimum sum edge coloring for the corresponding graph $G^{\prime}$. In particular, $G$ and $G^{\prime}$ have the same edge strength. In Section 6, we show that for every $k \geq 3$, it is $\Theta_{2}^{p}$-complete to determine whether the edge strength of a quasigraph is at most $k$. By the above construction, $\Theta_{2^{-}}^{p}$ completeness follows for ordinary simple graphs as well.

Gadgets. The reduction in Section 6 is of the component design type: we build "gadgets" corresponding to vertices and edges, and in the reduction a larger graph is constructed from these smaller graphs. In some cases, these gadgets themselves are also built from smaller gadgets. Here we introduce the terminology and the notational conventions that will be used while working with gadgets.

A gadget is a graph whose vertices are divided into external and internal vertices. On the figures, the external vertices of the gadgets are framed (see, for example, Figure 6.1 or Figure 6.2). If an external vertex has degree one, then the edge incident to it will be called a pendant edge (for example, the gadget in Figure 6.1 has 3 pendant edges).

We will use two operations to create larger graphs from smaller components. If $u$ and $v$ are vertices of $G$ and $H$, respectively, then the two gadgets can be joined by identifying these two vertices (see Figure 3.1b). In particular, if $v$ is the end vertex of a pendant edge $g$, then this operation will be called attaching the pendant edge $g$ of $H$ to vertex $u$ of $G$. If $e$ is a pendant edge of $G$, and $f$ is a pendant edge of $H$, then we can form a larger gadget by identifying these two edges (see Figure 3.1c). 


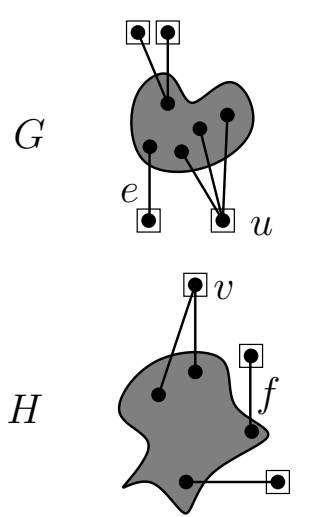

(a)

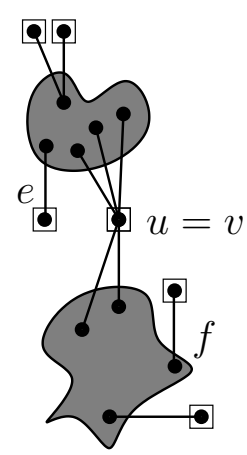

(b)

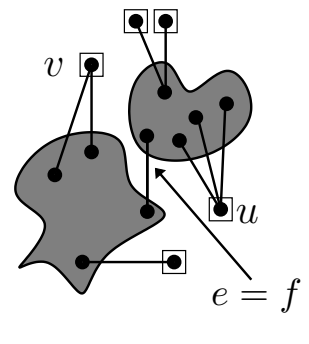

(c)

Figure 3.1: The two different ways of combining gadgets. (a) Two gadgets $G$ and $H$. (b) Identifying the vertices $u$ and $v$. (c) Identifying the two edges $e$ and $f$.

\section{Graphs with $s^{\prime}(G)>\chi^{\prime}(G)$}

The aim of this section is to show that for every $k \geq 3$, there is a simple graph $G$ with $\Delta(G)=\chi^{\prime}(G)=k$ and $s^{\prime}(G)=k+1$. This gives a counterexample to the conjecture of Harary and Plantholt (see West Winter 1994-95) for every possible value of $k$. Notice that for $k=2$ there are no such graphs: if $\chi^{\prime}(G)=2$, then every connected component of $G$ is a path or an even cycle, which can be edge colored optimally with 2 colors.

It turns out that for $k>3$, the graphs constructed by Izbicki (1964) (long before the conjecture) have the required properties. For every $k \geq 3$, the Izbicki graph $I_{k}\left(V_{k}, E_{k}\right)$ is defined as follows (see Figure 4.1):

$$
\begin{aligned}
V_{k} & =\left\{R_{s}, Q_{t}, P_{t} \mid 1 \leq s \leq k-3,1 \leq t \leq k\right\}, \\
E_{k} & =\left\{\left(R_{s}, Q_{t}\right),\left(Q_{t}, Q_{t+1}\right),\left(Q_{t}, P_{t}\right) \mid 1 \leq s \leq k-3,1 \leq t \leq k\right\},
\end{aligned}
$$

where $Q_{k+1}=Q_{1}$. We note that these graphs were used by Leven \& Galil (1983) to reduce the edge coloring problem of multigraphs to the edge coloring of simple graphs. Vertices $R_{s}$ and $Q_{t}$ have degree $k$, while vertices $P_{t}$ have degree 1 ; therefore, by the following lemma, the $k$ edges $\left(Q_{t}, P_{t}\right)$ have pairwise different colors in every $k$-edge-coloring of $I_{k}$.

Lemma 4.1 (Izbicki 1964). Let $G$ be a graph that contains only degree 1 and degree $k$ vertices; denote by $n$ the number of vertices in $G$ having degree $k$, 

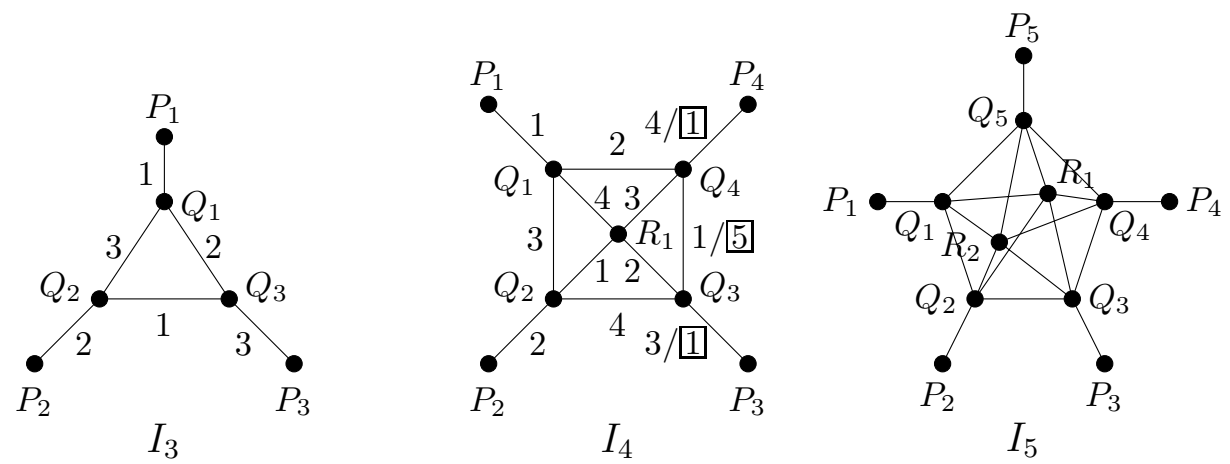

Figure 4.1: Izbicki's graphs for $k=3,4,5$. In $I_{4}$, the sum of the coloring decreases if we use the colors shown in frames.

and let $F$ be the set of edges incident to the degree 1 vertices. In every $k$-edgecoloring of $G$, if $f_{i}(1 \leq i \leq k)$ denotes the number of edges in $F$ with color $i$, then $f_{i}$ has the same parity as $n$.

Proof. If vertex $v$ has degree $k$, then every color appears at $v$ in every $k$ edge-coloring. Therefore, with the above notation, color $i$ appears at exactly $n+f_{i}$ vertices. This number must be even, hence $n$ and $f_{i}$ have the same parity.

Since $I_{k}$ has $n=2 k-3$ vertices with degree $k$, thus the Lemma implies that $f_{i}$ is odd for every $1 \leq i \leq k$. The set $F$ contains $k$ edges in the graph $I_{k}$, hence every $f_{i}$ is 1 , and the edges in $F$ have pairwise different colors. Therefore, if $I_{k}$ has a $k$-edge-coloring, then this coloring has error $\sum_{i=1}^{k}(i-1)=k(k-1) / 2$, since the degree $k$ vertices $R_{s}, Q_{t}$ have zero error. Moreover, $I_{k}$ is $k$-edgecolorable, as shown by the following coloring $\psi$ :

$$
\begin{aligned}
\psi\left(R_{s}, Q_{t}\right) & =[t+s+2]_{k}(1 \leq s \leq k-3,1 \leq t \leq k) \\
\psi\left(Q_{t}, P_{t}\right) & =t(1 \leq t \leq k) \\
\psi\left(Q_{t}, Q_{t+1}\right) & =[t+2]_{k}(1 \leq t \leq k)
\end{aligned}
$$

where $[x]_{k}=x-k$ for $x>k$, and $[x]_{k}=x$ for $x \leq k$.

Now consider the coloring $\psi^{\prime}$ that is the same as $\psi$ except that

$$
\begin{aligned}
\psi^{\prime}\left(Q_{k-1}, P_{k-1}\right) & =1 \text { instead of } k-1 \\
\psi^{\prime}\left(Q_{k-1}, Q_{k}\right) & =k+1 \text { instead of } 1 \\
\psi^{\prime}\left(Q_{k}, P_{k}\right) & =1 \text { instead of } k .
\end{aligned}
$$




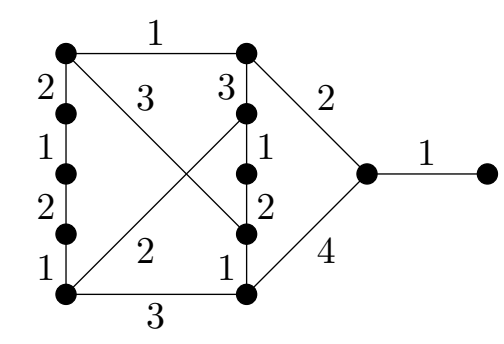

Figure 4.2: A graph with $\Delta(G)=\chi^{\prime}(G)=3$ but $s^{\prime}(G)=4$. The figure shows a minimum sum edge coloring with sum 29 using 4 colors, every 3-edge-coloring has sum at least 30 .

This modification increases the sum by $(1+(k+1)+1)-((k-1)+1+k)=3-k$, which is negative if $k>3$. Therefore, $I_{k}$ (for $k>3$ ) has a $(k+1)$-edge-coloring with sum strictly smaller than the minimum sum that can be achieved by any $k$-edge-coloring, hence $s^{\prime}\left(I_{k}\right)>k=\chi^{\prime}\left(I_{k}\right)=\Delta\left(I_{k}\right)$.

Proposition 4.2. For every $k>3, \chi^{\prime}\left(I_{k}\right)=k$ and $s^{\prime}\left(I_{k}\right)=k+1$.

For $k=3$, the graph $I_{3}$ does not provide a counterexample to the conjecture of Harary and Plantholt, as the minimum sum 12 can be achieved using only 3 colors (see Figure 4.1). However, the 3-edge-colorable graph shown in Figure 4.2 gives a counterexample for the case $k=3$. This graph is the smallest counterexample for $k=3$, and was found by an exhaustive computerized search. The search was performed using the program nauty of Brendan McKay (see McKay 1990), which is capable of enumerating all non-isomorphic graphs with a given number of vertices and maximum degree. For each graph it was first checked whether it is 3-edge-colorable, and if so, then the sum of the best 3-edge-coloring and the best 4-edge-coloring was determined by a simple backtracking method. Checking all the 19430 non-isomorphic connected graphs on 12 vertices with maximum degree 3 took under a minute on a $800 \mathrm{MHz}$ computer.

Figure 4.2 shows a 4-edge-coloring of the graph with sum 29. Unfortunately, we cannot give a hand-verifiable proof that this sum cannot be achieved by a 3edge-coloring. However, a very simple program can check all the $3^{15} \approx 14.3 \cdot 10^{6}$ possible 3 -edge-colorings of the 15 edges, and can verify that the best 3-edgecoloring has sum 30.

Proposition 4.3. For every $k \geq 3$, there is a simple graph $G_{k}$ with $\Delta\left(G_{k}\right)=$ $\chi^{\prime}\left(G_{k}\right)=k$ and $s^{\prime}\left(G_{k}\right)=k+1$. 


\section{The complexity class $\Theta_{2}^{p}$}

In the introduction, we have argued that the problem of deciding whether $s^{\prime}(G) \leq k$ holds does not seem to belong to either NP or coNP. Thus determining the chromatic edge strength of a graph seems to be a problem more difficult than those contained in NP. However, not much more difficult: using an NP-oracle, the value of $\Sigma^{\prime}(G)$ can be determined with a polynomial number of oracle queries, and with one additional query it can be decided whether there is a $k$-edge-coloring with sum $\Sigma^{\prime}(G)$. In fact, a logarithmic number of oracle queries is sufficient: the value of $\Sigma^{\prime}(G)$ can be determined using binary search. Therefore, as an upper bound, it can be said that this problem is in $\mathrm{P}^{\mathrm{NP}}=\Delta_{2}^{p}$. But exactly where does this problem lie between NP and $\Delta_{2}^{p}$ ?

The class $\Theta_{2}^{p}=\mathrm{P}^{\mathrm{NP}[O(\log n)]}$ contains those languages that can be decided by a polynomial-time oracle Turing-machine that makes $O(\log n)$ adaptive queries to an NP oracle. There are several other natural characterizations of $\Theta_{2}^{p}$ in the literature: as shown in Hemachandra (1989); Papadimitriou \& Zachos (1982); Wagner (1990), it is equivalent to $\mathrm{P}_{\|}^{\mathrm{NP}}$ (polynomial-time computation with parallel access to an NP-oracle), $\mathrm{L}_{\|}^{\mathrm{NP}}$ (log-space bounded computation with parallel access to an NP-oracle), and $\mathrm{L}^{\mathrm{NP}}$ (log-space computation with an NPoracle). The notation $\Theta_{2}^{p}$ comes from Wagner (1990), who defines this class as part of the polynomial hierarchy: $\Theta_{i+1}^{p}$ is the class of problems that can be decided in polynomial time by at most $O(\log n)$ queries to a $\Sigma_{i}^{p}$-oracle. Clearly, $\Sigma_{i}^{p}, \Pi_{i}^{p} \subseteq \Theta_{i+1}^{p} \subseteq \Delta_{i+1}^{p}$. It is conjectured that these inclusions are proper. However, our present knowledge does not even rule out the possibility of $\mathrm{P}=$ PSPACE.

There are some more exotic characterizations of $\Theta_{2}^{p}$. For example, Lange \& Reinhardt (1994) introduced the concept of empty alternation, and proved the surprising result that log-space and polynomial-time bounded computation with auxiliary Turing tape and empty alternation equals $\Theta_{2}^{p}$. Holzer \& McKenzie (2003) gave similar characterizations of $\Theta_{2}^{p}$ using auxiliary stacks (see also Holzer \& McKenzie 2002).

The class $\Theta_{2}^{p}$ turns out to be relevant in other ways as well. Mahaney (1982) has shown that if NP has a sparse Turing-complete set, then the polynomial hierarchy (PH) collapses to $\Delta_{2}^{p}$. Kadin (1989) has strengthened this result by showing that if NP has sparse Turing-complete sets, then $\mathrm{PH} \subseteq \Theta_{2}^{p}$. Moreover, this theorem is optimal in the sense that the collapse to $\Theta_{2}^{p}$ relativizes, but there are relativized worlds with sparse NP-complete sets where $\mathrm{PH}$ does not collapse bellow $\Theta_{2}^{p}$ (Kadin 1989).

If a complexity class has several natural complete problems, then this makes 
the class natural and worth studying. The abundance of complete problems is usually taken as a sign that the class captures some important aspect of computation. Wagner (1987) has shown that NP-hard optimization problems often give rise to $\Theta_{2}^{p}$-complete decision problems. For example, it is $\Theta_{2}^{p}$-complete to decide if the size of a maximum independent set in $G$ is odd, or to decide if two graphs $G_{1}$ and $G_{2}$ have maximum independent sets of the same size.

Besides these somewhat technical problems, $\Theta_{2}^{p}$ has more natural complete problems. The following greedy algorithm is a well-known heuristic for the maximum independent set problem: take a vertex with minimum degree, put it into the independent set, delete it and its neighbors from the graph, and continue this while there are vertices in the graph. In general, this will not necessarily result in a maximum independent set, but in certain graphs, with a lucky sequence of choices, it is possible that the result is optimal. Hemaspaandra \& Rothe (1998) showed that it is $\Theta_{2}^{p}$-complete to decide whether the greedy algorithm can find a maximum independent set in the given graph $G$. More generally, for every rational number $r \geq 1$, they show that it is $\Theta_{2}^{p}$-complete to decide whether the greedy algorithm can find an $r$-approximation of the optimum, that is, an independent set of size at least $1 / r$ times the maximum.

Another example can be found in the study of electoral systems. The Condorcet Paradox states that even if every voter has a clear preference order of the candidates, it is not necessary that there is a "best" candidate who can beat every other candidate in pairwise comparisons (such a candidate is called a Condorcet winner). In 1876 Lewis Caroll proposed an electoral system that can be used to find a winner even if there is no such best candidate: let that candidate be the winner who can become a Condorcet winner with a minimal number of changes in the preferences of the voters. Hemaspaandra et al. (1997) showed that it is $\Theta_{2}^{p}$-complete to decide whether candidate $\mathrm{X}$ is the winner in this system. It is quite fascinating to see that there is a $\Theta_{2}^{p}$-complete problem that was posed more than 100 years before the definition of the class $\Theta_{2}^{p}$.

In Section 6, we show that for every $k \geq 3$, it is $\Theta_{2}^{p}$-complete to decide whether $s^{\prime}(G) \leq k$ holds. In order to prove this result, we introduce four new $\Theta_{2}^{p}$-complete variants of the minimum vertex cover problem:

\section{Minimum Vertex Cover with $\hat{v}$}

Input: A graph $G(V, E)$ and a distinguished vertex $\hat{v} \in V$

Question: [Does at least one/Does every] minimum vertex cover in $G$ [contains/avoids] $\hat{v}$ ? 
The four problems are abbreviated MVC- $\exists \in$, MVC- $\exists \notin$, MVC- $\forall \in$, MVC$\forall \notin$ (the symbol $\exists$ stands for "Does at least one," $\forall$ stands for "Does every," $\in$ stands for "contains," and $\notin$ stands for "avoids"). The rest of this section is devoted to the proof that these four problems are $\Theta_{2}^{p}$-complete for 3-regular graphs. This result might be of independent interest as well. In the following, we denote by $\tau(G)$ the size of the minimum vertex cover of $G$.

LEMma 5.1. The MVC- $\exists \in$ problem is $\Theta_{2}^{p}$-hard.

Proof. Given two graphs $G_{1}$ and $G_{2}$, it is $\Theta_{2}^{p}$-complete to decide whether $\tau\left(G_{1}\right) \leq \tau\left(G_{2}\right)$ holds (Spakowski \& Vogel 2000). We reduce this decision problem to MVC- $\exists \in$. Add a new isolated vertex $\hat{v}$ to $G_{2}$, let $G_{2}^{\prime}$ be the resulting graph. We can assume that $G_{1}$ and $G_{2}^{\prime}$ have the same number $n$ of vertices: otherwise we could add new isolated vertices without changing the problem. Let $G$ be the graph obtained by joining every vertex of $G_{1}$ with every vertex of $G_{2}^{\prime}$. A vertex cover $S$ of $G$ has to contain either every vertex of $G_{1}$ or every vertex of $G_{2}^{\prime}$ : if a vertex $u \in G_{1}$ is missing from $S$, then every neighbor of $u$ has to be in $S$. Therefore, a minimum vertex cover of $G$ either

1. contains every vertex of $G_{1}$ and a minimum vertex cover of $G_{2}^{\prime}$, or

2. contains every vertex of $G_{2}^{\prime}$ and a minimum vertex cover of $G_{1}$.

In the first case the size of the vertex cover is $n+\tau\left(G_{2}^{\prime}\right)=n+\tau\left(G_{2}\right)$, in the second case $n+\tau\left(G_{1}\right)$. If $\tau\left(G_{1}\right)>\tau\left(G_{2}\right)$, then every minimum vertex cover is of the first type, otherwise there is at least one minimum vertex cover of the second type. Thus if $\tau\left(G_{1}\right) \leq \tau\left(G_{2}\right)$, then there is a minimum vertex cover that contains $\hat{v}$ (second type), otherwise there is no such minimum vertex cover.

Theorem 5.2. All four problems MVC- $\exists \in$, MVC- $\exists \notin$, MVC- $\forall \in$, MVC- $\forall \notin$ are $\Theta_{2}^{p}$-complete for 3-regular graphs.

Proof. To see that these problems belong to the class $\Theta_{2}^{p}$, observe that by using binary search, a logarithmic number of adaptive NP-oracle calls are sufficient to determine $\tau(G)$, the size of the minimum vertex cover in the graph. Having done that, a single NP or coNP query can answer whether there is a vertex cover, whether every vertex cover of size $\tau(G)$ has the required property.

Lemma 5.1 proves that the MVC- $\exists \in$ problem is $\Theta_{2}^{p}$-hard. We show that the problem remains $\Theta_{2}^{p}$-hard when restricted to 3-regular graphs. Given a graph $G$ with a distinguished vertex $\hat{v}$, we transform it to a graph $G^{\prime}$ with maximum 
degree 3 without changing the answer to the MVC- $\exists \in$ problem. Later we will transform this graph into a 3-regular graph $G^{\prime \prime}$.

Let $v$ be a vertex of $G$, and consider an arbitrary ordering of the neighbors of $v$. The vertex $v$ is replaced by a cycle $C_{v}$ of length $2 d(v)$ having vertices $a_{v, 1}, b_{v, 1}, \ldots, a_{v, d(v)}, b_{v, d(v)}$. If $u$ and $v$ are neighbors in $G$, then $C_{u}$ and $C_{v}$ are connected by an edge. More precisely, if $u$ is the $i$-th neighbor of $v$, and $v$ is the $j$-th neighbor of $u$, then vertex $a_{v, i}$ and $a_{u, j}$ are neighbors in $G^{\prime}$. Furthermore, for every vertex $v$ in $G$, a degree 1 vertex $c_{v}$ is attached to $b_{v, 1}$. This completes the description of $G^{\prime}$.

We claim that $\tau\left(G^{\prime}\right)=\sum_{v \in V} d(v)+\tau(G)$. Let $X_{v}=\left\{a_{v, 1}, b_{v, 1}, \ldots, a_{v, d(v)}\right.$, $\left.b_{v, d(v)}, c_{v}\right\}$. The $X_{v}$ 's form a partition of the vertex set of $G^{\prime}$. Since $X_{v}$ contains a cycle of length $2 d(v)$, every vertex cover has to contain at least $d(v)$ vertices from $X_{v}$. Thus the size of every vertex cover in $G^{\prime}$ is at least $\sum_{v \in V} d(v)$. Furthermore, if a vertex cover contains exactly $d(v)$ vertices from $X_{v}$, then these vertices have to be $b_{v, 1}, \ldots, b_{v, d(v)}$. Therefore, if $u$ and $v$ are neighbors in $G$, then every vertex cover in $G^{\prime}$ has to contain either more than $d(u)$ vertices from $X_{u}$ or more than $d(v)$ vertices from $X_{v}$, otherwise the edge connecting $X_{u}$ and $X_{v}$ would not be covered. This implies that in at least $\tau(G)$ of the $X_{v}$ 's, the vertex cover contains more than $d(v)$ vertices, hence the size of every vertex cover in $G^{\prime}$ is at least $\sum_{v \in V} d(v)+\tau(G)$. On the other hand, if $S$ is a vertex cover of $G$, then we can construct a vertex cover of $G^{\prime}$ as follows. For each $v \in S$, we add the vertices $\left\{a_{v, 1}, \ldots, a_{v, d(v)}, c_{v}\right\}$ to the vertex cover; for $v \notin S$, we add the vertices $\left\{b_{v, 1}, \ldots, b_{v, d(v)}\right\}$. This is gives a vertex cover of $G^{\prime}$ having size exactly $\sum_{v \in V} d(v)+\tau(G)$.

Let $a_{\hat{v}, 1}$ be the distinguished vertex of $G^{\prime}$. If there is a minimum vertex cover of $G$ that contains $\hat{v}$, then the argument in the previous paragraph shows that there is a minimum vertex cover of $G^{\prime}$ that contains $a_{\hat{v}, 1}$. Conversely, assume that $S^{\prime}$ is a minimum vertex cover of $G^{\prime}$. Let $S$ be the set of those $v$ 's for which $S$ contains more than $d(v)$ vertices of $X_{v}$. As we have seen above, $S$ is a minimum vertex cover of $G$. From $a_{\hat{v}, 1} \in S^{\prime}$ it follows that $\hat{v} \in S$, hence in this case $G$ has a minimum vertex cover containing the distinguished vertex $\hat{v}$.

The same reduction can be used to show the $\Theta_{2}^{p}$-completeness of MVC- $\exists \notin$ for graphs with maximum degree 3 . This time let $b_{\hat{v}, 1}$ be the distinguished vertex of $G^{\prime}$. A similar argument shows that $G$ has a minimum vertex cover containing $\hat{v}$ if and only if $G^{\prime}$ has a minimum vertex cover not containing $b_{\hat{v}, 1}$, hence MVC- $\exists \notin$ has the same complexity as MVC- $\exists \in$. Moreover, since MVC- $\forall \in$ is the complement of MVC- $\exists \notin$, and MVC- $\forall \notin$ is the complement of MVC- $\exists \in$, it follows that the remaining two problems are $\Theta_{2}^{p}$-complete as well, because $\Theta_{2}^{p}$ is closed under taking complements. 


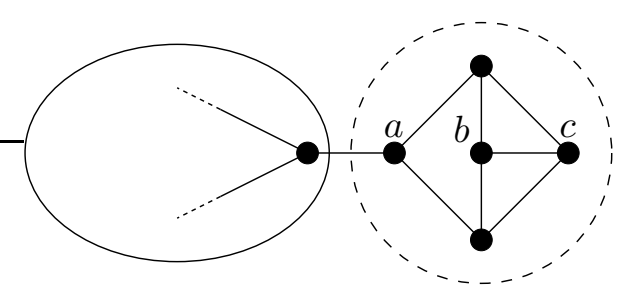

Figure 5.1: Attaching gadgets to the graph to make it 3-regular.

The theorem requires us to prove the $\Theta_{2}^{p}$-completeness of the problems for 3-regular graphs, but the constructed graph $G^{\prime}$ has vertices with degree less than 3. If a vertex $v$ of $G^{\prime}\left(V^{\prime}, E^{\prime}\right)$ has degree less than 3 , then attach to $v$ one or two gadgets to make the degree of $v$ exactly 3, Figure 5.1 shows the attached gadget. Assume that $g$ such gadgets are attached, denote by $G^{\prime \prime}\left(V^{\prime \prime}, E^{\prime \prime}\right)$ the resulting 3-regular graph. At least 3 vertices of each gadget have to be selected to cover the edges of the gadgets, thus $\tau\left(G^{\prime \prime}\right) \geq \tau\left(G^{\prime}\right)+3 g$. On the other hand, given a vertex cover of $G^{\prime}$, adding to this set the vertices $a, b, c$ of every gadget yields a vertex cover of $G^{\prime \prime}$; therefore, $\tau\left(G^{\prime \prime}\right)=\tau\left(G^{\prime}\right)+3 g$. Moreover, every minimum vertex cover of $G^{\prime}$ can be extended to a minimum vertex cover of $G^{\prime \prime}$, and if $S$ is a minimum vertex cover of $G^{\prime \prime}$, then $S \cap V^{\prime}$ induces a minimum vertex cover of $G^{\prime}$. Therefore, every minimum vertex cover of $G^{\prime}$ contains the distinguished vertex if and only if every minimum vertex cover of $G^{\prime \prime}$ contains it, hence the theorem is proved for 3-regular graphs as well.

Replacing minimum vertex cover with maximum independent set in the problem definition results in four new problems MIS- $\exists \in$, MIS- $\exists \notin$, MIS- $\forall \in$, MIS- $\forall \notin$. From the well-known fact that every maximum independent set is the complement of a minimum vertex cover, it follows that these four problems are equivalent to the four problems MVC- $\exists \notin$, MVC- $\exists \in$, MVC- $\forall \notin$, MVC- $\forall \in$, respectively. For example, there is a maximum independent set containing vertex $\hat{v}$ if and only if there is a minimum vertex cover not containing $\hat{v}$.

Corollary 5.3. All four problems MIS- $\exists \in$, MIS- $\exists \notin$, MIS- $\forall \in$, MIS- $\forall \notin$ are $\Theta_{2}^{p}$-complete for 3-regular graphs.

\section{The reduction}

For every $k \geq 3$, we prove that it is $\Theta_{2}^{p}$-complete to decide whether $s^{\prime}(G) \leq k$ holds for a given graph $G$. The reduction is from the MVC- $\exists \in$ problem defined in Section 5. Given a 3-regular graph $G$, we construct a quasigraph $G^{\prime}$ such 


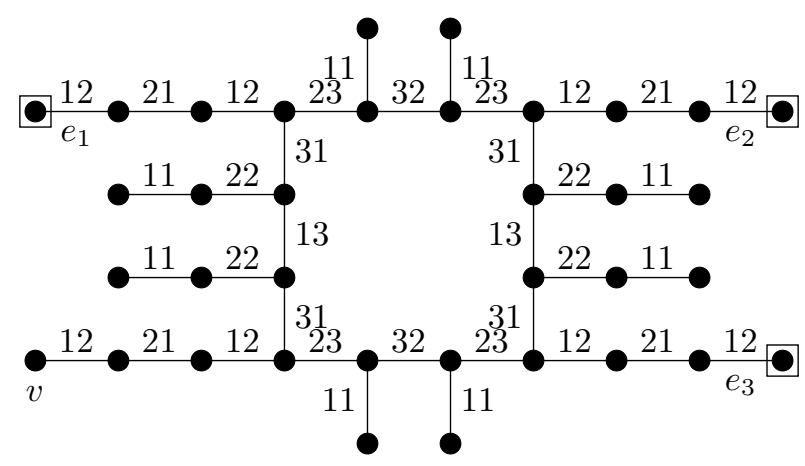

Figure 6.1: The vertex gadget. The two numbers on each edge show two edge colorings of the gadget.

that $\epsilon\left(G^{\prime}\right)=\tau(G)+c_{k}$, where $\tau(G)$ is the size of a minimum vertex cover in $G$, and $c_{k}$ is a constant depending only on $k$. Moreover, the minimum error $\tau(G)+c_{k}$ can be achieved by a $k$-edge-coloring of $G^{\prime}$ if and only if there is a minimum vertex cover of $G$ containing the distinguished vertex $\hat{v}$. This means that $s^{\prime}(G) \leq k$ if and only if the answer to the MVC- $\exists \in$ problem is yes. The constructed graph $G^{\prime}$ is a quasigraph, but we want to prove that checking $s^{\prime}(G) \leq k$ is $\Theta_{2}^{p}$-hard for simple graphs. However, this is not a problem, as the transformation of Proposition 3.1 gives us a simple graph $G^{\prime \prime}$ with the same edge strength as $G^{\prime}$.

The quasigraph $G^{\prime}$ is constructed by associating vertex gadgets and edge gadgets to the vertices and edges of $G$. The vertex gadget shown in Figure 6.1 has 3 pendant edges that correspond to the 3 edges incident to the vertex in $G$. The coloring of the pendant edges will determine whether we add the vertex to the vertex cover or not. If the vertex is in the vertex cover, then all 3 pendant edges are colored with color 2, otherwise the pendant edges have color 1 . The gadget has the following properties:

- There is a coloring $\psi$ with zero error on the internal vertices of the vertex gadget that colors all three pendant edges with color 1. Moreover, every coloring with zero error on the internal vertices colors the pendant edges with color 1 .

- There is a coloring $\psi^{*}$ that colors all three pendant edges with color 2 and has an error of 1 on the internal vertices.

Figure 6.1 shows two edge colorings of the vertex gadget. The first coloring has zero error on the internal vertices and assigns color 1 to the pendant edges. The 


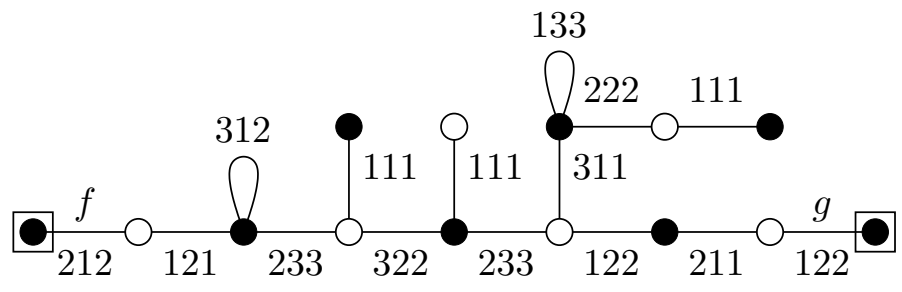

Figure 6.2: The edge gadget with 3 different edge colorings.

error of the second coloring is 1 (the error occurs at vertex $v$ ), and assigns color 2 to the pendant edges. Moreover, the first coloring is the unique coloring with zero error on the internal vertices: the reader can easily verify this by observing that the edges incident to degree 1 vertices have to be colored with color 1 , and the implications of this uniquely determines the coloring of the rest of the graph. These observations prove that the gadget has the required properties.

The edge gadget shown in Figure 6.2 has two pendant edges $f$ and $g$. If a coloring has zero error on the internal vertices of the gadget, then clearly edges $f$ and $g$ have color 1 or 2 . There are 4 different ways of coloring $f$ and $g$ with these two colors. In 3 out of 4 of these combinations, when at least one of $f$ and $g$ is colored with color 2 , the coloring can be extended to the whole gadget with zero error (Figure 6.2 shows these 3 different colorings). On the other hand, if both $f$ and $g$ have color 1 , then there is at least one error on the internal vertices of the gadget. The reader can verify this by following the implications of coloring $f$ and $g$ with color 1 , and requiring that every internal vertex has zero error.

For the distinguished vertex $\hat{v}$, a more complicated gadget is required than the vertex gadget shown in Figure 6.1. Like the vertex gadget, the special vertex gadget has a low-error coloring that assigns color 1 to the three pendant edges, and there is a coloring with error greater by 1 that assigns color 2 to the pendant edges. Furthermore, the low-error coloring can be achieved only with $\Delta+1$ colors, while the other coloring uses only $\Delta$ colors. The following lemma states the properties of this gadget formally:

LEMMA 6.1 (Special vertex gadget). For every $k \geq 3$, if there is a simple graph $H_{k}$ with $\chi^{\prime}\left(H_{k}\right)=\Delta\left(H_{k}\right)=k$ and $s^{\prime}\left(H_{k}\right)=k+1$, then there is a quasigraph $D_{k}$ satisfying the following requirements:

(i) $D_{k}$ has three pendant edges. Denote by $V_{0}$ the internal vertices of $D_{k}$.

(ii) Every edge coloring $\psi$ with $\epsilon_{\psi}\left(V_{0}\right)=\epsilon\left(V_{0}\right)$ uses at least $k+1$ colors and assigns color 1 to the three pendant edges. 
(iii) There is a $(k+1)$-edge-coloring $\psi$ with $\epsilon_{\psi}\left(V_{0}\right)=\epsilon\left(V_{0}\right)$ that assigns color 1 to the three pendant edges.

(iv) There is a $k$-edge-coloring $\psi^{*}$ with $\epsilon_{\psi^{*}}\left(V_{0}\right)=\epsilon\left(V_{0}\right)+1$ that assigns color 2 to the three pendant edges.

The proof of Lemma 6.1 is deferred to Section 7. We note here that the vertex gadget of Figure 6.1 satisfies these properties with $\epsilon\left(V_{0}\right)=0$, except for Property (ii).

Theorem 6.2. For every $k \geq 3$, if there is a graph $H_{k}$ with $\chi^{\prime}\left(H_{k}\right)=\Delta\left(H_{k}\right)=$ $k$ and $s^{\prime}\left(H_{k}\right)=k+1$, then it is $\Theta_{2}^{p}$-complete to decide whether $s^{\prime}(G) \leq k$.

Proof. The proof is by reduction from the MVC- $\exists \in$ problem, which was proved $\Theta_{2}^{p}$-complete in Section 5 (Theorem 5.2). Given a 3-regular graph $G(V, E)$ with a distinguished vertex $\hat{v}$, we construct a quasigraph $G^{\prime}$ with maximum degree $k$ such that $s^{\prime}(G)=k$ if and only if there is a minimum vertex cover of $G$ containing $\hat{v}$.

The quasigraph $G^{\prime}\left(V^{\prime}, E^{\prime}\right)$ is constructed as follows. Initially, let us ignore the distinguished vertex $\hat{v}$, considering it an ordinary vertex like the others. Later we will show what modifications have to be done for $\hat{v}$. For each vertex $v$ of $G$, a vertex gadget $S_{v}$ is added to $G^{\prime}$, and for each edge $e$ of $G$, an edge gadget $S_{e}$ is added to $G^{\prime}$. Direct the edges of $G$ arbitrarily. For each vertex $v$ of $G$, consider an arbitrary ordering of the 3 edges incident to $v$. If the $i$-th edge incident to $v \in V(i=1,2,3)$ is an edge $e$ entering $v$, then identify pendant edge $e_{i}$ of $S_{v}$ with pendant edge $f$ of $S_{e}$. If the $i$-th edge incident to $v \in V$ is an edge $e$ leaving $v$, then identify edge $e_{i}$ of $S_{v}$ with edge $g$ of $S_{e}$. Thus every vertex of $G^{\prime}$ is an internal vertex of a vertex gadget $S_{v}$ or an edge gadget $S_{e}$. Denote by $V_{v}$ the internal vertices of gadget $S_{v}$ and by $V_{e}$ the internal vertices of $S_{e}$, by construction these sets form a partition of $V^{\prime}$.

We claim that $G^{\prime}$ has an edge coloring with error $t$ if and only if $G$ has a vertex cover of size $t$. Assume first that $D \subseteq V$ is a vertex cover of $G$. If $v \in D$, then color gadget $S_{v}$ using coloring $\psi^{*}$ : every pendant edge has color 2 and there is an error of 1 on the internal vertices. If $v \notin D$, then we use coloring $\psi$ of the vertex gadget: every pendant edge of $S_{v}$ has color 1 and there is no error on the internal vertices. Now consider a gadget $S_{e}$ for some $e \in E$. The two pendant edges $f$ and $g$ are already colored with color 1 or 2 . However, at least one of them is colored with 2 , since at least one end vertex of $e$ is in $D$. Therefore, using one of the three colorings shown in Figure 6.2, we can extend the coloring to every edge of $S_{e}$ with zero error on the internal vertices of the 
gadget. Errors appear on the internal vertices of $S_{v}$ only for $v \in D$, hence the total error of the coloring is $|D|$.

On the other hand, consider an edge coloring of $G^{\prime}$ with error $t$. Let $\widehat{V} \subseteq V$ be the set of those $v \in V$ for which there is error in $V_{v}$. Similarly, let $\widehat{E} \subseteq E$ be the set of those $e \in E$ for which there is error in $V_{e}$. Clearly, the coloring has error at least $|\widehat{V}|+|\widehat{E}| \leq t$. Let $\bar{V}$ be a set of $|\widehat{E}|$ vertices in $G$ that cover every edge in $\widehat{E}$. The set of vertices $\widehat{V} \cup \bar{V}$ has size at most $|\widehat{V}|+|\widehat{E}| \leq t$. We show that this set is a vertex cover of $G$. It is clear that every edge $e \in \widehat{E}$ is covered, since there is a $v \in \bar{V}$ covering $e$. Now consider an edge $e \notin \widehat{E}$, this means that $V_{e}$ is colored with zero error; thus, as we have observed, at least one pendant edge of $S_{e}$ is colored with color 2 . If this edge is the pendant edge of the vertex gadget $S_{v}$, then there is at least one error in $V_{v}$ and $v$ is in $\widehat{V}$. However, if the pendant edge of $S_{e}$ and $S_{v}$ is identified in the construction, then $e$ is incident to $v$, and it follows that $v \in \widehat{V}$ covers $e$.

We have shown that $\epsilon\left(G^{\prime}\right)=\tau(G)$. Now we modify $G^{\prime}$ slightly to take into account the distinguished vertex $\hat{v}$. The gadget corresponding to vertex $\hat{v}$ is not the vertex gadget of Figure 6.1, but the special vertex gadget defined in Lemma 6.1. By modifying appropriately the argument presented above, one can show that $\epsilon\left(G^{\prime}\right)=\tau(G)+\epsilon\left(V_{0}\right)$, where $\epsilon\left(V_{0}\right)$ is the minimum error on the internal vertices of the special gadget. Moreover, if $G$ has a minimum vertex cover $D$ containing $\hat{v}$, then $G^{\prime}$ has a minimum sum edge coloring using only $k$ colors, since in this case we can use the coloring $\psi^{*}$ on the special gadget. On the other hand, if there is a minimum sum edge coloring using $k$ colors, then by Property (ii) of Lemma 6.1, the error is more than $\epsilon\left(V_{0}\right)$ on the internal vertices of the special gadget. This means that vertex $\hat{v}$ is contained in the set $\widehat{V}$ defined above, hence the constructed minimum vertex cover contains $\hat{v}$. Therefore, $s^{\prime}\left(G^{\prime}\right)=k$ if and only if $G$ has a minimum vertex cover containing $\hat{v}$, what we had to prove.

In Section 4 we have seen that for every $k \geq 3$, there is a simple graph with maximum degree and chromatic index $k$ that has edge strength $k+1$. Combining this with Theorem 6.2 gives

Corollary 6.3. For every fixed $k \geq 3$, it is $\Theta_{2}^{p}$-complete to decide whether $s^{\prime}(G) \leq k$.

We note that deciding $s^{\prime}(G) \leq 2$ is trivial: $s^{\prime}(G) \leq 2$ is only possible if $\chi^{\prime}(G) \leq 2$, which means that every connected component of the graph is a path or an even cycle. It is easy to see that $s^{\prime}(G)=\chi^{\prime}(G)=2$ holds for every such graph. 
Determining the chromatic edge strength is the special case of determining the chromatic strength: edge strength of $G$ is simply the strength of the line graph of $G$. Therefore, Corollary 6.3 implies hardness for the chromatic strength as well:

Corollary 6.4. For every fixed $k \geq 3$, it is $\Theta_{2}^{p}$-complete to decide whether $s(G) \leq k$.

In the introduction, we have noted that if $G$ is a simple graph, then $s^{\prime}(G)$ is either $\Delta(G)$ or $\Delta(G)+1$ (Hajiabolhassan et al. 2000; Mitchem et al. 1997), and consequently, $s^{\prime}(G)$ is either $\chi^{\prime}(G)$ or $\chi^{\prime}(G)+1$. In Theorem 6.2 , we construct a graph with maximum degree and chromatic index $k$. Therefore, comparing $s^{\prime}(G)$ to $\Delta(G)$ or to $\chi^{\prime}(G)$ is also hard:

Corollary 6.5. For every $k \geq 3$, it is $\Theta_{2}^{p}$-complete to decide for graphs with maximum degree $k$ whether $s^{\prime}(G)=\Delta(G)$ holds.

Corollary 6.6. For every $k \geq 3$, it is $\Theta_{2}^{p}$-complete to decide for graphs with maximum degree $k$ whether $s^{\prime}(G)=\chi^{\prime}(G)$ holds.

Hajiabolhassan et al. (2000) asked for a characterization of those graphs where $s^{\prime}(G) \neq \chi^{\prime}(G)$. Corollary 6.6 implies that we cannot hope for a nontrivial (NP or coNP) characterization of such graphs.

\section{Special vertex gadget}

The aim of this section is to construct the special vertex gadget defined in Lemma 6.1. However, some preparations are required before the proof. We recursively construct two families of trees $T_{i}$ and $N_{i}(i \geq 1)$. Every $T_{i}$ has a pendant edge $e$, and every $N_{i}$ has a root $r$. The trees $T_{1}$ and $N_{1}$ consist of a single edge. The tree $T_{i}$ is the same as $N_{i-1}$, with a pendant edge connected to the root $r$. The tree $N_{i}$ is constructed by attaching the pendant edges of a $T_{1}, T_{2}, \ldots, T_{i}$ tree to a common root $r$. The construction is demonstrated in Figure 7.1 .

The properties of these trees are summarized in the following lemma:

LEmma 7.1. (a) There is an edge coloring of the tree $T_{i}$ that has no error on the internal vertices of $T_{i}$, and assigns color $i$ to the pendant edge $e$. Furthermore, every coloring that assigns color $j$ to $e$ has error at least $|j-i|$ on the internal vertices. 

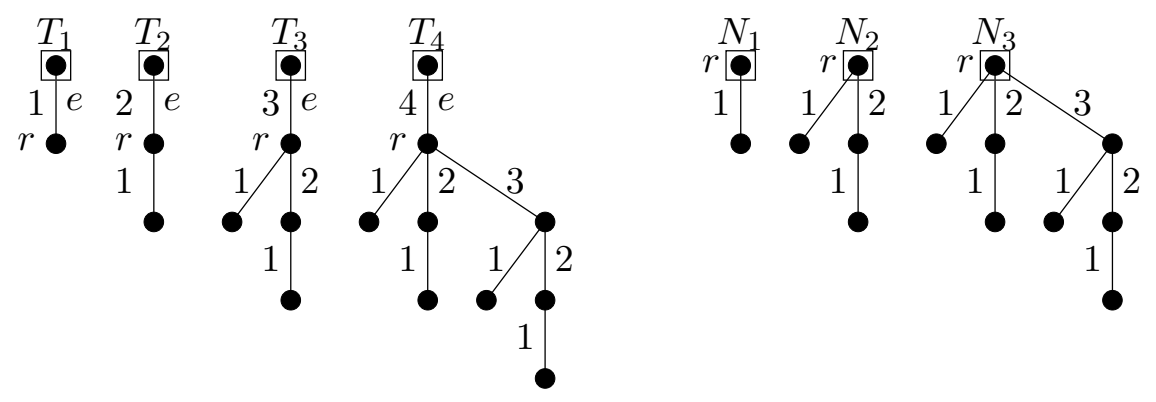

Figure 7.1: The trees $T_{i}$ and $N_{i}$.

(b) There is a zero error edge coloring of the tree $N_{i}$ that assigns the colors $1,2, \ldots, i$ to the edges incident to $r$. Furthermore, if color $j \leq i$ is missing at $r$ in a coloring, then this coloring has error at least $i-j+1$ on the internal vertices of $N_{i}$.

Proof. The proof is by induction on $i$. Both statements are trivial for $i=1$. Now assume that $i>1$ and both (a) and (b) hold for every $1 \leq i^{\prime}<i$. First we prove statement (a). Since $T_{i}-e$ is isomorphic to $N_{i-1}$, it has a zero error coloring by the induction hypotheses. Extending this coloring by assigning color $i$ to edge $e$ does not create errors on the internal vertices of $T_{i}$, proving the first part of statement (a). Consider now an edge coloring of $T_{i}$ that assigns color $j$ to $e$. This coloring colors $T_{i}-e=N_{i-1}$ in such a way that color $j$ is missing at vertex $r$. If $j<i$, then by the induction hypothesis, there is an error of at least $(i-1)-j+1=|j-i|$ on the internal vertices of $N_{i-1}$, and we are done. On the other hand, if $j>i$, then in the coloring of $T_{i}$ the degree $i$ internal vertex $r$ has error at least $j-i$.

Next we prove statement (b). Let $e_{1}, e_{2}, \ldots, e_{i}$ be the edges incident to $r$ in $N_{i}$, edge $e_{j}$ is the pendant edge of the tree $T_{j}$ attached to $r$. A zero error edge coloring of $N_{i}$ can be obtained by coloring every attached tree $T_{j}$ in such a way that the internal vertices have zero error and edge $e_{j}$ has color $j$. Clearly, there is no error on $r$ or on any other vertex of $N_{i}$ in this coloring.

Suppose that a color $j \leq i$ is missing from $r$ in a coloring $\psi$ of $N_{i}$. Define the following sequence of edges: $e_{s_{1}}=e_{j}$ and $e_{s_{k+1}}=e_{\psi\left(e_{s_{k}}\right)}$ until an edge with $\psi\left(e_{s_{k^{\prime}}}\right)>i$ is found (it can be verified that this sequence is finite). Since $e_{s_{k}}$ is the pendant edge of a tree $T_{s_{k}}$, by statement (a), there is error at least $\left|s_{k}-\psi\left(e_{s_{k}}\right)\right|$ on the internal vertices of $T_{s_{k}}$. Therefore, the internal vertices of 
$N_{i}$ have error at least

$$
\begin{array}{r}
\left|\psi\left(e_{s_{1}}\right)-s_{1}\right|+\left|\psi\left(e_{s_{2}}\right)-s_{2}\right|+\cdots+\left|\psi\left(e_{s_{k^{\prime}-1}}\right)-s_{k^{\prime}-1}\right|+\left|\psi\left(e_{s_{k^{\prime}}}\right)-s_{k^{\prime}}\right| \\
\geq\left(\psi\left(e_{s_{1}}\right)-s_{1}\right)+\left(\psi\left(e_{s_{2}}\right)-s_{2}\right)+\cdots+\left(\psi\left(e_{s_{k^{\prime}-1}}\right)-s_{k^{\prime}-1}\right)+\left(\psi\left(e_{s_{k^{\prime}}}\right)-s_{k^{\prime}}\right) \\
=\psi\left(e_{s_{k^{\prime}}}\right)-s_{1} \geq i+1-j,
\end{array}
$$

since by definition, $\psi\left(e_{s_{k}}\right)=s_{k+1}$ for $1 \leq k<k^{\prime}$, and $\psi\left(e_{s_{k^{\prime}}}\right)>i$.

The coloring defined by Lemma 7.1 will be called the standard coloring of these gadgets. In the standard coloring of $T_{i}$ the pendant edge receives color $i$, and the color of every other edge is less than $i$. Moreover, the tree $T_{i}$ can be colored such that the pendant edge has color $j$ and the internal error is exactly $|i-j|$. To see this, consider the standard coloring of $T_{i}$, and recolor the pendant edge with color $j$. If $j>i$, then this results in a proper coloring with internal error $j-i$. If $j<i$, then the recolored pendant edge will conflict with an edge $f$ that has color $j$ in the standard coloring. The conflict can be resolved by giving color $i$ to $f$ (this does not cause any further conflicts, since in the standard coloring only the pendant edge has color $i$ ). The recoloring introduces an error of $i-j$ at one end point of $f$.

Denote by $\Sigma_{\Delta}^{\prime}(G)$ the minimum sum that a $\Delta(G)$-edge-coloring of $G$ can have. By definition, $\Sigma_{\Delta}^{\prime}(G) \geq \Sigma^{\prime}(G)$. Denote by $\epsilon_{\Delta}(G)$ the error of the best $\Delta(G)$-edge-coloring, that is, $\epsilon_{\Delta}(G)=2 \Sigma_{\Delta}^{\prime}(G)-\ell(G)$.

In the following lemma, we determine how the errors on the vertices change if we attach an edge of $G_{2}$ to vertex $v$ of $G_{1}$.

LEMmA 7.2. Let $G_{1}\left(V_{1}, E_{1}\right)$ and $G_{2}\left(V_{2}, E_{2}\right)$ be two graphs such that $V_{1} \cap V_{2}=$ $\{v\}$ and edge $e$ is the only edge in $G_{2}$ incident to $v$. Let $d$ be the degree of $v$ in $G_{1}$. Let $G\left(V_{1} \cup V_{2}, E_{1} \cup E_{2}\right)$ be the graph obtained by joining $G_{1}$ and $G_{2}$ at vertex $v$. If $\psi_{1}$ is an edge coloring of $G_{1}, \psi_{2}$ is an edge coloring of $G_{2}$, and these colorings assign distinct colors to the edges incident to $v$, then they can be combined to obtain an edge coloring $\psi$ of $G$ such that

$$
\epsilon_{\psi}(u)= \begin{cases}\epsilon_{\psi_{1}}(u) & \text { if } u \in V_{1} \backslash\{v\} \\ \epsilon_{\psi_{2}}(u) & \text { if } u \in V_{2} \backslash\{v\} \\ \epsilon_{\psi_{1}}(u)+\psi_{2}(e)-(d+1) & \text { if } u=v\end{cases}
$$

Conversely, if $\psi$ is an edge coloring of $G$, then it induces an edge coloring $\psi_{1}$ of $G_{1}$ such that

$$
\epsilon_{\psi_{1}}(u)= \begin{cases}\epsilon_{\psi}(u) & \text { if } u \in V_{1} \backslash\{v\} \\ \epsilon_{\psi}(v)-\psi(e)+d+1 & \text { if } u=v\end{cases}
$$


Proof. The first statement clearly holds for every vertex $u \neq v$, since combining the two colorings can change the error only on $v$, the only common vertex of the two graphs. Let $E_{v} \subseteq E_{1}$ be the edges incident to $v$ in $G_{1}$. The error of $v$ in coloring $\psi$ is

$$
\begin{aligned}
\epsilon_{\psi}(v) & =\sum_{f \in E_{v}} \psi(f)+\psi(e)-\sum_{i=1}^{d+1} i=\sum_{f \in E_{v}} \psi_{1}(f)+\psi_{2}(e)-\sum_{i=1}^{d} i-(d+1) \\
& =\left(\sum_{f \in E_{v}} \psi_{1}(f)-\sum_{i=1}^{d} i\right)+\psi_{2}(e)-(d+1)=\epsilon_{\psi_{1}}(v)+\psi_{2}(e)-(d+1) .
\end{aligned}
$$

The second statement can be proved by a similar calculation.

In particular, if we attach a tree $T_{d(v)}$ to a vertex $v$, then the error changes as follows:

LEMmA 7.3. Let $v$ be an arbitrary vertex of the simple graph $G(V, E)$; attach to $v$ the pendant edge $e$ of the tree $T_{d(v)}$. Denote by $G^{\prime}$ the resulting graph.

(a) The error $\epsilon\left(G^{\prime}\right)$ is either $\epsilon(G)-1$ or $\epsilon(G)+1$, and it is $\epsilon(G)-1$ if and only if there is a minimum sum edge coloring $\psi$ of $G$ such that some color $c \leq d(v)$ is missing from $v$.

(b) If $d(v)<\Delta(G)$, then $\epsilon_{\Delta}\left(G^{\prime}\right)$ is either $\epsilon_{\Delta}(G)-1$ or $\epsilon_{\Delta}(G)+1$, and it is $\epsilon_{\Delta}(G)-1$ if and only if there is a $\Delta(G)$-edge-coloring with error $\epsilon_{\Delta}(G)$ where some color $c^{\prime} \leq d(v)$ is missing from $v$.

Proof. Let $\psi$ be a minimum sum edge coloring of $G$, and let $c \leq d(v)+1$ be the smallest color not present at $v$ in $\psi$. As discussed after the proof of Lemma 7.1, the tree $T_{d(v)}$ has a coloring that assigns color $c$ to the pendant edge $e$ and has internal error $|d(v)-c|$. This coloring can be combined with $\psi$ to obtain a coloring $\psi^{\prime}$ of $G^{\prime}$. We use Lemma 7.2 to calculate the error of $\psi^{\prime}$. The total error on the internal vertices of $T_{d(v)}$ is $|d(v)-c|$, and the error on the vertices of $G$ is the same as in $\psi$, except on $v$, where the error is increased by $c-(d(v)+1)$. Therefore, the error of $\psi^{\prime}$ is $\epsilon_{\psi^{\prime}}\left(G^{\prime}\right)=\epsilon_{\psi}(G)+c-(d(v)+$ $1)+|d(v)-c|$. If $c \leq d(v)$, then this equals $\epsilon_{\psi}(G)-1$, thus $\epsilon\left(G^{\prime}\right) \leq \epsilon(G)-1$. If $c=d(v)+1$, then $\epsilon_{\psi^{\prime}}(G)=\epsilon_{\psi}(G)+1$, and $\epsilon\left(G^{\prime}\right) \leq \epsilon(G)+1$ follows. Therefore, we have obtained that $\epsilon\left(G^{\prime}\right) \leq \epsilon(G)+1$, and if $G$ has a minimum sum edge coloring where a color $c \leq d(v)$ is missing from $v$, then $\epsilon\left(G^{\prime}\right) \leq \epsilon(G)-1$.

To finish the proof of statement (a), we have to show that $\epsilon\left(G^{\prime}\right) \geq \epsilon(G)-1$, and if every minimum sum edge coloring of $G$ uses at $v$ every color not greater than $d(v)$, then $\epsilon\left(G^{\prime}\right) \geq \epsilon(G)+1$. Assume that a minimum sum coloring $\psi^{\prime}$ of 
$G^{\prime}$ is given with $\psi^{\prime}(e)=c$. By Lemma 7.1, the error is at least $|d(v)-c|$ on the internal vertices of the tree $T_{d}(v)$, so the error, $\epsilon_{\psi^{\prime}}(V)$, is at most $\epsilon\left(G^{\prime}\right)-|d(v)-c|$ on the vertices $V$. The coloring $\psi^{\prime}$ induces a coloring $\psi$ of $G$, and by the second part of Lemma 7.2,

$\epsilon_{\psi}(G)=\epsilon_{\psi^{\prime}}(V)-c+d(v)+1 \leq \epsilon\left(G^{\prime}\right)-|d(v)-c|-c+d(v)+1 \leq \epsilon\left(G^{\prime}\right)+1$,

hence $\epsilon\left(G^{\prime}\right) \geq \epsilon_{\psi}(G)-1 \geq \epsilon(G)-1$. Moreover, equality is only possible if $c \leq d(v)$ and $\psi$ is a minimum sum edge coloring of $G$, or in other words, if there is a minimum sum edge coloring of $G$ such that color $c \leq d(v)$ is missing from $v$. Finally, if every minimum sum coloring of $G$ uses only colors at most $d(v)$ on $v$, then either $c>d(v)$ or $\psi$ is not a minimum sum coloring of $G$. In either case, $\epsilon_{\psi^{\prime}}\left(G^{\prime}\right) \geq \epsilon(G)+1$ follows, completing the proof of statement (a) (recall that if $\psi$ is not a minimum sum coloring of $G$, then $\epsilon_{\psi}(G) \geq \epsilon(G)+2$, since the error of every coloring has the same parity). The proof of statement (b) is exactly the same. Notice that if $d(v)<\Delta(G)$, then $\Delta\left(G^{\prime}\right)=\Delta(G)$.

The following gadget will be used in the construction of the special vertex gadget.

LEMma 7.4. For every $k \geq 1$, there is a quasigraph $H_{k}$ satisfying the following properties ( $V_{0}$ denotes the internal vertices of $H_{k}$ ):

(i) $H$ has two pendant edges $f, g$.

(ii) There is a $(k+1)$-edge-coloring $\psi_{k+1}$ with $\psi_{k+1}(f)=k+1, \psi_{k+1}(g)=1$ and $\epsilon_{\psi_{k+1}}\left(V_{0}\right)=0$.

(iii) For every $i \leq k$, there is a $(k+1)$-edge-coloring $\psi_{i}$ with $\psi_{i}(f)=i, \psi_{i}(g)=$ 2 , and $\epsilon_{\psi_{i}}\left(V_{0}\right)=k-i$.

(iv) For every coloring $\psi$, if $\psi(f)=i \leq k$, then $\epsilon_{\psi}\left(V_{0}\right) \geq k-i$.

(v) For every coloring $\psi$, if $\psi(f)=k+1$ and $\epsilon_{\psi}\left(V_{0}\right)=0$, then $\psi(g)=1$.

Proof. For $k=1,2,3$, the graph $H_{k}$ is shown in Figure 7.2. It can be verified directly that they satisfy the requirements of the lemma. For the remainder of the proof, it is assumed that $k \geq 4$.

The graph $H_{k}$ is constructed as follows. Take a path on 6 vertices $v_{1}, v_{2}$, $v_{3}, v_{4}, v_{5}, v_{6}$, let $f=v_{1} v_{2}$ and $g=v_{5} v_{6}$. Identify the root of a tree $N_{k-1}$ with vertex $v_{2}$. Attach a half-loop to $v_{3}$, and attach to $v_{3}$ the pendant edges of $k-2$ trees $T_{2}, T_{3}, \ldots, T_{k-1}$. Attach a half-loop to $v_{4}$ as well, and attach to $v_{4}$ the 


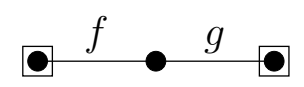

$H_{1}$

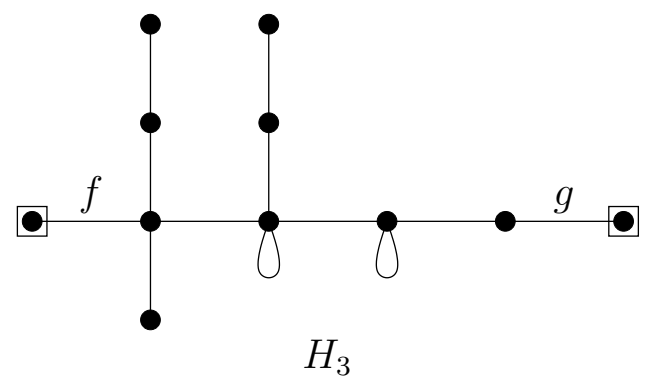

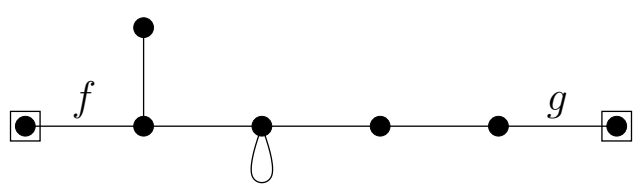

$\mathrm{H}_{2}$

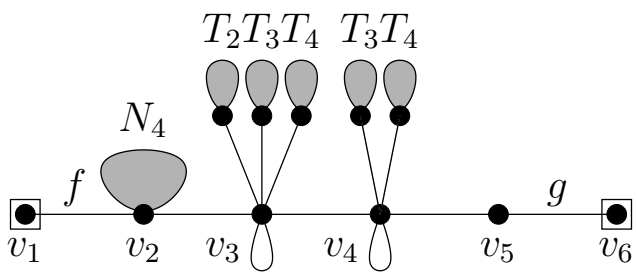

$H_{5}$

Figure 7.2: The graphs $H_{1}, H_{2}, H_{3}$, and $H_{5}$.

pendant edges of $k-3$ trees $T_{3}, T_{4}, \ldots, T_{k-1}$. The resulting graph $H_{k}$ is shown in Figure 7.2.

The coloring $\psi_{k+1}$ is defined by $\psi_{k+1}\left(v_{1} v_{2}\right)=k+1, \psi_{k+1}\left(v_{2} v_{3}\right)=k$, $\psi_{k+1}\left(v_{3} v_{4}\right)=1, \psi_{k+1}\left(v_{4} v_{5}\right)=2, \psi_{k+1}\left(v_{5} v_{6}\right)=1, \psi_{k+1}\left(v_{3} v_{3}\right)=k+1, \psi_{k+1}\left(v_{4} v_{4}\right)=$ $k$, and it gives the standard coloring to the attached trees. It can be verified that $\psi_{k+1}$ is a proper edge coloring and there is zero error on the internal vertices, which gives Property (ii). Similarly, the coloring $\psi_{k}$ required by Property (iii) for $i=k$ is defined as $\psi_{k}\left(v_{1} v_{2}\right)=k, \psi_{k}\left(v_{2} v_{3}\right)=k+1, \psi_{k}\left(v_{3} v_{4}\right)=k$, $\psi_{k}\left(v_{4} v_{5}\right)=1, \psi_{k}\left(v_{5} v_{6}\right)=2, \psi_{k}\left(v_{3} v_{3}\right)=1, \psi_{k}\left(v_{4} v_{4}\right)=2$, with the standard coloring on the attached trees.

To obtain the coloring $\psi_{i}$ for some $i<k$ (Property (iii)), take the coloring $\psi_{k}$ defined above, and exchange the colors $k$ and $i$ on the alternating path starting at edge $f$. (An alternating path is a path where the colors of the edges are $k$ and $i$ alternately. There is a unique maximal alternating path starting from $f$.) Exchanging $k$ and $i$ on the path introduces an error of $k-i$ at a single vertex, namely the vertex at the other end of the alternating path. Notice that this color exchange cannot affect edge $g$, since edge $v_{2} v_{3}$ has color $k+1$. Therefore, we obtain a coloring satisfying Property (iii).

To see that Property (iv) holds, observe that a coloring $\psi$ of $H_{k}$ induces a coloring of the tree $N_{k-1}$, and color $\psi(f)$ is missing from the root of $N_{k-1}$. 
Therefore, in this coloring of $N_{k-1}$, there is error at least $k-1-\psi(f)+1=k-i$ on the internal vertices (Lemma 7.1b), and this means that $\psi$ has error at least $k-i$ on the internal vertices of $H_{k}$, as required.

To verify Property $(\mathrm{v})$, assume that $\psi(f)=k+1$ and $e_{\psi}\left(V_{0}\right)=0$, that is, there is zero error on each internal vertex of the gadget. The color of edge $v_{2} v_{3}$ cannot be less than $k$, since in that case the tree $N_{k-1}$ could not be colored with zero error on its internal vertices. Vertex $v_{2}$ has degree $k+1$, hence the assumption that there is no error on $v_{2}$ implies that $\psi\left(v_{2} v_{3}\right) \leq k+1$. Color $k+1$ is used by $f$ on $v_{2}$, therefore we can conclude that $\psi\left(v_{2} v_{3}\right)=k$. For $2 \leq i \leq k-1$, edge $v_{3} v_{4}$ cannot have color $i$, since that would imply that the tree $T_{i}$ attached to vertex $v_{3}$ cannot be colored with zero internal error. Since vertex $v_{4}$ has degree $k$, and color $k$ is already used at $v_{3}$ by edge $v_{2} v_{3}$, it follows that $\psi\left(v_{3} v_{4}\right)=1$. This implies in turn that $\psi\left(v_{4} v_{5}\right) \neq 1$. However, there is zero error on vertex $v_{5}$; therefore, there must be an edge with color 1 at $v_{5}$. Thus edge $g$ has color 1 , as required.

Now we are ready to construct the special vertex gadget:

Proof (Proof of Lemma 6.1). By assumption, there exists a graph $G$ with $\Delta(G)=k$ and $s^{\prime}(G)=k+1$ (or equivalently, $\epsilon(G)<\epsilon_{\Delta}(G)$ ). If more than one graph satisfies this condition, then select a graph $G$ such that

$$
\epsilon_{\Delta}(G)-\epsilon(G)>0 \text { is minimal, }
$$

and among these graphs,

$$
\epsilon(G) \text { is minimal. }
$$

For every vertex $v$ of $G$, we define two sets $\Lambda(v), \Lambda_{\Delta}(v) \subseteq\{1,2, \ldots, d(v)\}$. Set $\Lambda(v)$ contains $j(1 \leq j \leq d(v))$ if there is an edge coloring of $G$ with error $\epsilon(G)$ such that $j$ is missing from $v$. If $\Lambda(v)=\emptyset$, then this means that every minimum sum edge coloring has zero error on $v$. Similarly, $\Lambda_{\Delta}(v)$ contains $j$ $(1 \leq j \leq d(v))$, if there is a $\Delta(G)$-edge-coloring with error $\epsilon_{\Delta}(G)$ such that $j$ is missing from $v$.

First we show that at least one of $\Lambda(v)$ and $\Lambda_{\Delta}(v)$ is empty for every vertex $v$. Otherwise attach the pendant edge of a tree $T_{d(v)}$ to $v$, let $G^{\prime}$ be the resulting graph. Since there are colors $j \in \Lambda(v), j^{\prime} \in \Lambda_{\Delta}(v)$ not greater than $d(v)$, by (a) and (b) of Lemma 7.3, we have $\epsilon\left(G^{\prime}\right)=\epsilon(G)-1$ and $\epsilon_{\Delta}\left(G^{\prime}\right)=\epsilon_{\Delta}(G)-1$, which contradicts the minimality of $G$ with respect to $(* *)$.

Since $\epsilon_{\Delta}(G)>0$, there is at least one vertex $v$ with $\Lambda_{\Delta}(v) \neq \emptyset, \Lambda(v)=\emptyset$. Call such a vertex a join vertex (later we will join another gadget to $G$ at such 


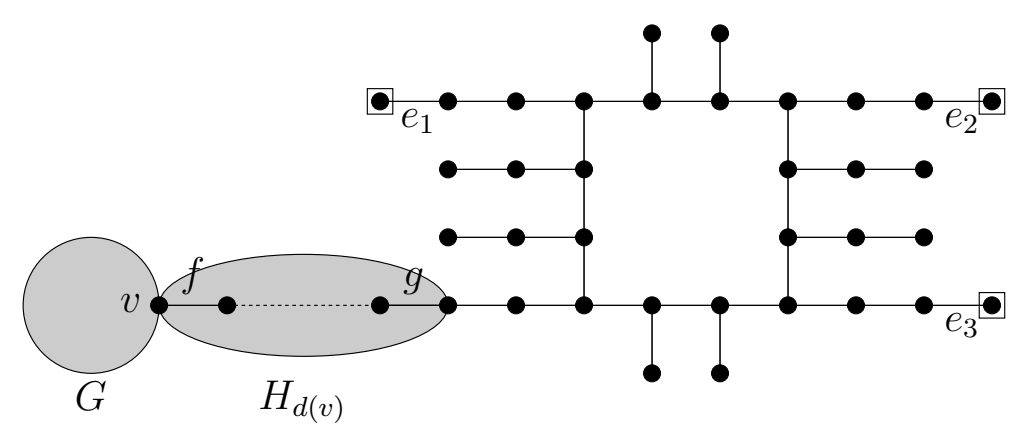

Figure 7.3: The structure of the special vertex gadget $D_{k}$.

a vertex, hence the name). Notice that $d(v)<\Delta(G)$, since $\Lambda_{\Delta}(v) \neq \emptyset$ means that there is a $\Delta(G)$-edge-coloring that uses a color greater than $d(v)$ at $v$.

The error has the same parity in every edge coloring, and $\epsilon_{\Delta}(G)>\epsilon(G)$ by assumption, thus it follows that $\epsilon_{\Delta}(G) \geq \epsilon(G)+2$. We claim that $\epsilon_{\Delta}(G)=$ $\epsilon(G)+2$ holds for a minimal graph $G$. Assume that on the contrary, $\epsilon_{\Delta}(G)>$ $\epsilon(G)+2$, and let $v$ be a join vertex in $G$. Attach to $v$ a tree $T_{d(v)}$ and let $G^{\prime}$ be the resulting graph. Since $\Lambda_{\Delta}(v) \neq \emptyset$, there is a $\Delta(G)$-edge-coloring of $G$ with error $\epsilon_{\Delta}(G)$ such that some color $c \leq d(v)$ is missing from $v$, thus by Lemma 7.3b, $\epsilon_{\Delta}\left(G^{\prime}\right)=\epsilon_{\Delta}(G)-1$. Moreover, since $\Lambda(v)=\emptyset$, every color not greater than $d(v)$ is used at $v$ in every minimum sum edge coloring of $G$, hence $\epsilon\left(G^{\prime}\right)=\epsilon(G)+1$, by Lemma 7.3a. Hence

$$
\epsilon_{\Delta}\left(G^{\prime}\right)-\epsilon\left(G^{\prime}\right)=\left(\epsilon_{\Delta}(G)-1\right)-(\epsilon(G)+1)=\epsilon_{\Delta}(G)-\epsilon(G)-2 .
$$

This value is larger than 0 by the assumption $\epsilon_{\Delta}(G)>\epsilon(G)+2$. Therefore, $G$ is not minimal with respect to $(*)$.

Now we are ready to construct the graph $D_{k}$. As shown in Figure 7.3, the graph $D_{k}$ consists of three parts: the minimal graph $G$ defined above, a graph $H_{i}$ from Lemma 7.4, and the variable gadget shown in Figure 6.1. Let vertex $v$ be a join vertex of $G$. Set $d=d(v)$, and connect to $v$ the pendant edge $f$ of graph $H_{d}$. Finally, as shown on the figure, a graph with 34 new vertices is connected to the pendant edge $g$ of $H_{d}$. The edges $e_{1}, e_{2}, e_{3}$ are the pendant edges of $D_{k}$.

Denote by $V_{0}$ the internal vertices of $D_{k}$ and let $V_{G}$ be the vertices of $G$ (including $v$ ).

Claim 7.5. If $V_{0}$ is the set of internal vertices of $D_{k}$, then $\epsilon\left(V_{0}\right)=\epsilon(G)$. Moreover, if $\epsilon_{\psi}\left(V_{0}\right)=\epsilon(G)$ for a coloring $\psi$ of $D_{k}$, then $\psi$ uses $\Delta(G)+1$ colors, $\psi(f)=d+1$ and $\psi\left(e_{i}\right)=1$ for $i=1,2,3$. 
Proof. Color $G$ with error $\epsilon(G)$ such that colors $1,2, \ldots, i$ appear at vertex $v$ (such a coloring exists, since $v$ is a join vertex and $\Lambda(v)=\emptyset$ ). Color the edges in $H_{d}$ using coloring $\psi_{d+1}$ of Lemma 7.4, it assigns color $d+1$ to $f$, and it does not introduce additional error on $v$ or on the internal vertices of $H_{i}$. Since this coloring assigns color 1 to edge $g$, it can be extended (in a unique way) to the rest of graph $D_{k}$ without increasing the error on $V_{0}$ (similarly as in the case of the vertex gadget of Section 6). Therefore, $\epsilon\left(V_{0}\right) \leq \epsilon(G)$. Notice that this coloring assigns color 1 to the edges $e_{1}, e_{2}, e_{3}$.

To show that $\epsilon\left(V_{0}\right) \geq \epsilon(G)$, let $\psi$ be an edge coloring of $D_{k}$ with $\epsilon_{\psi}\left(V_{0}\right) \leq$ $\epsilon(G)$. First we show that $\psi(f)>d$. If not, then by Property (iv) of Lemma 7.4, $\psi$ has error at least $d-\psi(f)$ on the internal vertices of $H_{d}$, hence there can be error at most $\epsilon_{\psi}\left(V_{0}\right)-(d-\psi(f)) \leq \epsilon(G)-(d-\psi(f))$ on $V_{G}$. By the second part of Lemma 7.2, this implies that $\psi$ induces a coloring $\psi^{\prime}$ of $G$ with error at most $\epsilon_{\psi^{\prime}}(G) \leq \epsilon_{\psi}\left(V_{G}\right)-\psi(f)+d+1 \leq \epsilon(G)-(d-\psi(f))-\psi(f)+d+1=\epsilon(G)+1$. Furthermore, $\psi^{\prime}$ is not a minimum sum edge coloring of $G$, since color $\psi(f) \leq d$ is missing from $v$, and $\Lambda(v)=\emptyset$. Therefore, $\epsilon_{\psi^{\prime}}(G)>\epsilon(G)$, but this also means that $\epsilon_{\psi^{\prime}}(G) \geq \epsilon(G)+2$, since the parity of the error is the same in every edge coloring. However, this contradicts $\epsilon_{\psi^{\prime}}(G) \leq \epsilon(G)+1$.

Therefore, it can be assumed that $\psi(f)>d$ for any coloring with $\epsilon_{\psi}\left(V_{0}\right) \leq$ $\epsilon(G)$. Now, again by Lemma 7.2, $\psi$ induces a coloring $\psi^{\prime}$ of $G$ with error

$$
\epsilon_{\psi^{\prime}}(G)=\epsilon_{\psi}\left(V_{G}\right)-\psi(f)+(d+1) \leq \epsilon_{\psi}\left(V_{G}\right) \leq \epsilon_{\psi}\left(V_{0}\right) \leq \epsilon(G)
$$

Since by definition $\epsilon_{\psi^{\prime}}(G) \geq \epsilon(G)$, these inequalities have to be equalities throughout. In particular, $\psi(f)=d+1$ and $\epsilon_{\psi}\left(V_{0}\right)=\epsilon(G)$, thus $\epsilon\left(V_{0}\right)$ cannot be strictly smaller than $\epsilon(G)$. Furthermore, every coloring $\psi$ with $\epsilon_{\psi}\left(V_{0}\right)=\epsilon(G)$ induces a coloring $\psi^{\prime}$ of $G$ with error $\epsilon(G)$. We know that error $\epsilon(G)$ can be achieved only by using $\Delta(G)+1$ colors. Therefore, $\Delta(G)+1$ colors are required to achieve error $\epsilon\left(V_{0}\right)=\epsilon(G)$ on $V_{0}$. Moreover, we have seen that in such a coloring $\psi$, the edge $f$ has color $d+1$ and the error on $V_{0} \backslash V$ is zero. By Property (v) of Lemma 7.4, this implies that $\psi(g)=1$ and it follows that the pendant edges $e_{1}, e_{2}, e_{3}$ also have color 1 , as required.

Property (ii) of Lemma 6.1 follows immediately from Claim 7.5. Moreover, in the proof of the claim we have constructed a coloring $\psi$ with $\epsilon_{\psi}\left(V_{0}\right)=\epsilon\left(V_{0}\right)$ and $\psi\left(e_{i}\right)=1$ for $i=1,2,3$, which implies Property (iii).

To show that Property (iv) holds, color $G$ using $\Delta(G)$ colors with error $\epsilon_{\Delta}(G)=\epsilon(G)+2$ such that color $c \in \Lambda_{\Delta}(v)$ is missing at vertex $v$; denote this coloring by $\psi_{\Delta}$. Color $H_{d}$ such that edge $f$ has color $c$, edge $g$ has color 2 , and there is error $d-c$ on the internal vertices of $H_{d}$ (the coloring $\psi_{c}$ from 
Property (iii) of Lemma 7.4). This coloring can be extended to a coloring of $D_{k}$ without introducing further errors on $V_{0}$ (see the second coloring in Figure 6.1), which gives a coloring $\psi^{*}$ that assigns color 2 to the three pendant edges $e_{1}, e_{2}, e_{3}$. We use the first part of Lemma 7.2 to determine $\epsilon_{\psi^{*}}\left(V_{0}\right)$. There is error $d-c$ on $V_{0} \backslash V$, and $\epsilon_{\psi^{*}}(u)=\epsilon_{\psi_{\Delta}}(u)$ for every $u \in V \backslash\{v\}$. By Lemma 7.2, $\epsilon_{\psi^{*}}(v)=\epsilon_{\psi_{\Delta}}(v)+\psi^{*}(f)-(d(v)+1)=\epsilon_{\psi_{\Delta}}(v)+c-d-1$. Therefore, $\epsilon_{\psi^{*}}\left(V_{0}\right)=\epsilon_{\psi_{\Delta}}(G)+(c-d-1)+(d-c)=\epsilon_{\Delta}(G)-1=\epsilon(G)+1=\epsilon\left(V_{0}\right)+1$, as required.

\section{Acknowledgements}

I'm grateful to Lane Hemaspaandra for valuable details on the history of the class $\Theta_{2}^{p}$ and for other useful comments. I'm very much indebted to the anonymous referees for their many remarks, which significantly improved the quality of the paper. In particular, they pointed out an error in the gadget construction of Theorem 2.2, and simplified the proof of Theorem 5.2. Research is supported in part by grants OTKA 44733, 42559, and 42706 of the Hungarian National Science Fund.

\section{References}

A. Bar-Noy, M. Bellare, M. M. Halldórsson, H. Shachnai \& T. Tamir (1998). On chromatic sums and distributed resource allocation. Inform. and Comput. 140(2), 183-202. ISSN 0890-5401.

A. Bar-Noy \& G. Kortsarz (1998). Minimum color sum of bipartite graphs. J. Algorithms 28(2), 339-365. ISSN 0196-6774.

M. Biró, M. Hujter \& Zs. Tuza (1992). Precoloring extension. I. Interval graphs. Discrete Math. 100(1-3), 267-279. ISSN 0012-365X.

H. L. Bodlaender, K. Jansen \& G. J. Woeginger (1994). Scheduling with incompatible jobs. Discrete Appl. Math. 55(3), 219-232. ISSN 0166-218X.

K. Giaro, R. Janczewski, M. Kubale \& M. Mazafiejski (2002). A 27/26Approximation Algorithm for the Chromatic Sum Coloring of Bipartite Graphs. In APPROX 2002, volume 2462 of Lecture Notes in Comput. Sci., 135-145. Springer, Berlin.

K. Giaro \& M. Kubale (2000). Edge-chromatic sum of trees and bounded cyclicity graphs. Inform. Process. Lett. 75(1-2), 65-69. ISSN 0020-0190. 
H. Hajiabolhassan, M. L. Mehrabadi \& R. Tusserkani (2000). Minimal coloring and strength of graphs. Discrete Math. 215(1-3), 265-270. ISSN 0012-365X.

M. M. Halldórsson, G. Kortsarz \& H. Shachnai (2003). Sum coloring interval and $k$-claw free graphs with application to scheduling dependent jobs. Algorithmica 37(3), 187-209. ISSN 0178-4617.

L. A. Hemachandra (1989). The strong exponential hierarchy collapses. J. Comput. System Sci. 39(3), 299-322. ISSN 0022-0000.

E. Hemaspandna, L. A. HemaspaAndra \& J. Rothe (1997). Exact analysis of Dodgson elections: Lewis Carroll's 1876 voting system is complete for parallel access to NP. J. ACM 44(6), 806-825. ISSN 0004-5411.

E. HemaspaAndra \& J. Rothe (1998). Recognizing when greed can approximate maximum independent sets is complete for parallel access to NP. Inform. Process. Lett. 65(3), 151-156. ISSN 0020-0190.

I. Holyer (1981). The NP-Completeness of Edge-Coloring. SIAM Journal on Computing 10(4), 718-720.

M. Holzer \& P. McKenzie (2002). On Auxiliary Pushdown and Stack Automata. ACM SIGACT News 33(1), 32-45. ISSN 0163-5700.

M. Holzer \& P. McKenzie (2003). Alternating and empty alternating auxiliary stack automata. Theoret. Comput. Sci. 299(1-3), 307-326. ISSN 0304-3975.

M. Hujter \& Zs. Tuza (1993). Precoloring Extension. II. Graph Classes Related To Bipartite Graphs. Acta Mathematica Universitatis Comenianae 62(1), 1-11.

H. IzBICKI (1964). An edge colouring problem. In Theory of Graphs and its Applications (Proc. Sympos. Smolenice, 1963), 53-61. Publ. House Czechoslovak Acad. Sci., Prague.

J. KAdin (1989). $\mathrm{P}^{\mathrm{NP}[O(\log n)]}$ and sparse Turing-complete sets for NP. J. Comput. System Sci. 39(3), 282-298. ISSN 0022-0000.

E. Kubicka (1989). The Chromatic Sum of a Graph. Ph.D. thesis, Western Michigan University.

E. Kubicka \& A. J. Schwenk (1989). An introduction to chromatic sums. In Proceedings of the ACM Computer Science Conf., 15-21. Springer, Berlin.

K.-J. Lange \& K. Reinhardt (1994). Empty alternation. In Mathematical Foundations of Computer Science 1994 (Košice, 1994), 494-503. Springer, Berlin. 
D. Leven \& Z. Galil (1983). NP Completeness of Finding the Chromatic Index of Regular Graphs. J. Algorithms 4(1), 35-44.

L. Lovász (1997). The membership problem in jump systems. J. Combin. Theory Ser. B 70(1), 45-66. ISSN 0095-8956.

S. R. Mahaney (1982). Sparse complete sets for NP: solution of a conjecture of Berman and Hartmanis. J. Comput. System Sci. 25(2), 130-143. ISSN 0022-0000.

D. Marx (2004). Precoloring extension on chordal graphs. Manuscript. Preliminary version presented at Graph Theory 2004, Paris.

D. MARx (2005). A short proof of the NP-completeness of minimum sum interval coloring. Oper. Res. Lett. 33(4), 382-384.

B. MCKAY (1990). Nauty user's guide, version 1.5. Technical Report TRCS-90-02, Computer Science Department, Australian National University. Also http://cs.anu.edu.au/people/bdm/nauty/.

J. Mitchem, P. Morriss \& E. Schmeichel (1997). On the cost chromatic number of outerplanar, planar, and line graphs. Discuss. Math. Graph Theory 17(2), 229241. ISSN 1234-3099.

S. Nicoloso, M. Sarrafzadeh \& X. Song (1999). On the sum coloring problem on interval graphs. Algorithmica 23(2), 109-126. ISSN 0178-4617.

C. H. Papadimitriou \& S. Zachos (1982). Two remarks on the power of counting. In Proceedings of the 6th GI-Conference on Theoretical Computer Science, 269-276. Springer-Verlag. ISBN 3-540-11973-6.

M. R. Salavatipour (2003). On sum coloring of graphs. Discrete Appl. Math. 127(3), 477-488. ISSN 0166-218X.

H. Spakowski \& J. Vogel (2000). $\Theta_{2}^{\mathrm{p}}$-completeness: a classical approach for new results. In FST TCS 2000: Foundations of software technology and theoretical computer science (New Delhi), volume 1974 of Lecture Notes in Comput. Sci., 348360. Springer, Berlin.

K. J. Supowit (1987). Finding a maximum planar subset of nets in a channel. IEEE Trans. Comput. Aided Design 6(1), 93-94.

T. Szkaliczki (1999). Routing with minimum wire length in the dogleg-free Manhattan model is NP-complete. SIAM J. Comput. 29(1), 274-287. ISSN 1095-7111.

Zs. Tuza (1997). Graph colorings with local constraints - a survey. Discuss. Math. Graph Theory 17(2), 161-228. ISSN 1234-3099. 
K. W. WaGner (1987). More complicated questions about maxima and minima, and some closures of NP. Theoret. Comput. Sci. 51(1-2), 53-80. ISSN 0304-3975.

K. W. Wagner (1990). Bounded query classes. SIAM J. Comput. 19(5), 833-846. ISSN 0097-5397.

D. West (Winter 1994-95). Open problems section. The SIAM Activity Group on Discrete Mathematics Newsletter 5(2), 9.

Manuscript received ?

DÁNIEL MARX

Department of Computer Science and Information Theory,

Budapest University of Technology and Economics

H-1521 Budapest, Hungary

dmarx@cs.bme.hu 